Submitted to the Annals of Statistics

\title{
SUPPLEMENTARY MATERIALS FOR: CAUSAL INFERENCE WITH A GRAPHICAL HIERARCHY OF INTERVENTIONS
}

In Appendix A, we give the rationale for the use of path interventions. In Appendix B, we list a few auxiliary definitions, and give arguments for our results on edge and path interventions. In addition, we link identifying functionals for certain responses to node interventions with identifying functionals for certain responses to edge interventions given by the edge g-formula.

Appendix A: Rationale for the Use of Path Interventions. We anticipate two possible objections to the use of path interventions. First, the inductive definition of responses to path interventions always defines them as nested counterfactuals where paths are not explicitly mentioned. For example, in Fig. 2 (a) in the paper, $Y\left((w A M Y)_{\rightarrow}\right) \equiv Y(M(A(w)), A)$. Therefore, it may seem that introducing an extra definition in terms of paths just introduces needlessly complicated syntax around an already known kind of object, a nested counterfactual. We call this the "syntactic objection."

Second, one of our main results is that path interventions are only identified under the MWM if they are expressible as edge interventions, and only identified under the SWM if they are expressible as node interventions. So an argument could be made that there is no particular reason to consider path interventions that are not also edge interventions, since responses to such are not identified under causal models in common use. We call this the "small ontology objection."

Our response to the syntactic objection is similar to the response made by members of the causal inference community that use graphical models. It is true that neither graphs, nor defining potential outcomes via paths is necessary for any mathematics that is being done. However, graphs are convenient for representing complex cause-effect relationships in an intuitive visual form. This helps make models and their assumptions transparent, and makes mathematical arguments about models easier to construct and to follow. Similarly, representing causal effects along particular pathways via a graphical notion of a path interventions, rather than via an algebraic notion of a nested potential outcome more closely corresponds to how people think about such effects intuitively. In our opinion, a response to a path intervention, with its associated syntax $Y\left((w A M Y)_{\rightarrow}\right)$, is a much more transparent representation of an effect of $W$ on $Y$ along the causal pathway $(W A M Y) \rightarrow$ 
in Fig. 2 (a) than a nested potential outcome with its associated syntax $Y(M(A(w)), A)$. The advantage of our representation is evident in our main result, which ties identifiability under particular causal models with graphical features with respect to which interventions are defined. An equivalent result could certainly be derived in algebraic language on nested potential outcomes such as $Y(M(A(w), A)$ directly, but would be much less understandable and more difficult to apply in practice.

Our response to the small ontology objection is that we do not believe operational considerations (in this case identifiability given a particular model) should guide how large our ontology (in this case the set of causal inference targets we are willing to entertain) should be. There are a number of reasons for this.

First, targets that happen not to be identifiable under a model we are willing to assume are often of substantive interest. For instance, unobserved confounding for total effects of interest often induces non-identifiability. This does not mean we should exclude total effects from consideration. Instead, we must find alternative ways to proceed, for instance by computing bounds on the effect. We give an example in section 4.3 of a seemingly innocuous version of a causal inference target popular in epidemiologic applications, the effect of treatment on the treated (with two time points) that can be expressed as a response to a path but not an edge intervention. Thus, if we were unwilling to consider path interventions, or an alternative representation of equivalent expressiveness, we would be unable to represent this type of target. Our results on path interventions also immediately imply this seemingly innocuous target is in fact not identified even under a strong causal model.

Second, a target that is not identified under a model may become identified under a submodel with more assumptions. For example, we give an example of a submodel of the MWM in section 5.4 where all responses to path interventions are identifiable. If we use identification to inform the size of our ontology, we are then placed in a strange situation where the set of objects we are willing to consider changes depending on what we are willing to assume in a particular problem.

Finally, we show that path interventions give us a general language for thinking about novel types of targets that generalize existing targets. In particular, in section 4.3 we discuss a new type of causal effect that combines features of the effect of treatment on the treated and path-specific effects. We argue that it would have been difficult to conceptualize such a target via a purely algebraic language. 


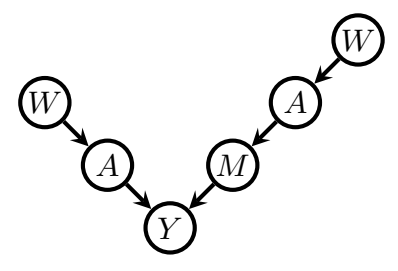

FIG 7. A defined response graph $\mathcal{F}(\{Y\},\{(W A Y) \rightarrow\}, \mathcal{G})$ for $\mathcal{G}$ in Fig. 2, representing a defined response $Y(A(w), M(A(W)))$ corresponding to $Y((w A Y) \rightarrow)$.

\section{Appendix B: Proofs and Auxiliary Definitions.}

Proofs for Section 3. To facilitate working with responses to path interventions, we introduce an alternative representation which makes certain results easier to prove.

A defined response of $Y$ to a path intervention $\pi$ is a counterfactual expression which results from fully applying the recursive substitution definition, and that only mentions ordinary (node) interventions. For example, a defined response corresponding to $Y\left((w A Y)_{\rightarrow}\right)$ in Fig. 2 is $Y(A(w), M(A(W)))$. Syntactically, a defined response for a single variable is a set of mentions of variables or values corresponding to variables in $\mathcal{G}$ (with a single variable or value possibly mentioned multiple times) arranged in an expression by means of comma separated lists enclosed by parentheses. For a mention $X$ in a defined response, we define the direct cause mentions to be the (possibly empty) set of mentions which occur to the right of $X$ in the expression and are separated from $X$ by exactly one unmatched left parenthesis. For instance, the direct cause mentions of the mention for $Y$ in $Y(A(w), M(A(W)))$ is the first mention of $A$, and the mention of $M$.

We give a graphical representation of responses corresponding to all mentions that occur in defined responses to path interventions, and the causal relationships among these responses as dictated by (4). Given a path intervention $\pi$ on $\boldsymbol{\alpha}$, and a set of outcomes $\mathbf{Y}$, let the defined response graph $\mathcal{F}(\mathbf{Y}, \boldsymbol{\alpha}, \mathcal{G})$ be a graph with a vertex for every mention which occurs in some defined response for $Y$, for each $Y \in \mathbf{Y}$, and a directed edge from $V_{1}$ to a $V_{2}$ if the mention corresponding to $V_{1}$ is a direct cause mention for the mention corresponding to $V_{2}$. Fig. 7 shows $\mathcal{F}\left(\{Y\},\left\{(W A Y)_{\rightarrow}\right\}, \mathcal{G}\right)$ for $\mathcal{G}$ in Fig 2 . We will call a parent-less node in $\mathcal{F}$ a leaf, and a childless node in $\mathcal{F}$ a root.

We give a number of results relating $\mathcal{F}(\mathbf{Y}, \boldsymbol{\alpha}, \mathcal{G})$ and $\mathcal{G}$. Those without proofs follow immediately by definition of recursive substitution and $\mathcal{F}$.

Lemma 0.1. If vertices $V_{1}, V_{2}$ in $\mathcal{F}(\mathbf{Y}, \boldsymbol{\alpha}, \mathcal{G})$ are in a parent-child rela- 
tionship, then so are (in $\mathcal{G})$ the vertices whose mentions $V_{1}, V_{2}$ correspond to.

Lemma $0.2 . \mathcal{F}(\mathbf{Y}, \boldsymbol{\alpha}, \mathcal{G})$ is a forest, that is each vertex $V_{1}$ in $\mathcal{F}$ has at most one child.

COROLlary 0.1. Every vertex in $\mathcal{F}(\mathbf{Y}, \boldsymbol{\alpha}, \mathcal{G})$ is a source of a directed path (possibly empty) to a root in $\mathcal{F}$, and no two vertices share such a path. Moreover, this directed path exists in $\mathcal{G}$.

Proof. That there is such a path and it is unique for each vertex follows by lemma 0.2 . The existence of a corresponding directed path in $\mathcal{G}$ follows by lemma 0.1 .

A vertex in $\mathcal{G}$ may correspond to multiple mentions in a given defined response, and thus to multiple vertices in $\mathcal{F}$, as is the case for $A$ in Fig. 7. However, there is a unique directed path in $\mathcal{G}$ for each vertex in $\mathcal{F}$ by corollary 0.1 . Thus, to differentiate vertices in $\mathcal{F}$ we will subscript mentions by their corresponding directed paths. For example, a node $A$ in $\mathcal{G}$ corresponds to nodes $A_{(A Y) \rightarrow}$, and $A_{(A M Y) \rightarrow}$ in $\mathcal{F}\left(\{Y\},\left\{(W A Y)_{\rightarrow}\right\}, \mathcal{G}\right)$ for $\mathcal{G}$ in Fig. 2 .

Lemma 0.3. If $W_{\alpha}$ is a leaf node in $\mathcal{F}(\mathbf{Y}, \boldsymbol{\alpha}, \mathcal{G})$, then either $\alpha$ contains a prefix subpath in $\boldsymbol{\alpha}$ or $\alpha$ does not contain any subpaths in $\boldsymbol{\alpha}$ and $\mathrm{pa}_{\mathcal{G}}(W)=\emptyset$.

Lemma 0.4. If $W_{\alpha} \in \mathcal{F}(\mathbf{Y}, \boldsymbol{\alpha}, \mathcal{G})$, then $\alpha \in \operatorname{rel}_{\mathcal{G}}(\mathbf{Y} \mid \boldsymbol{\alpha})$.

Proof. Fix a vertex $W_{\alpha}$ in $\mathcal{F}(\mathbf{Y}, \boldsymbol{\alpha}, \mathcal{G})$. That $\alpha$ is a directed path in $\mathcal{G}$ follows from lemma 0.1 .

Assume $W_{\alpha}$ is a leaf node in $\mathcal{F}(\mathbf{Y}, \boldsymbol{\alpha}, \mathcal{G})$. By lemma $0.3, \alpha \in \operatorname{rel}_{\mathcal{G}}(\mathbf{Y} \mid \boldsymbol{\alpha})$. If $W_{\alpha}$ is not a leaf node in $\mathcal{G}(\mathbf{Y}, \boldsymbol{\alpha}, \mathcal{G})$, then $\alpha$ is a suffix subpath of $\alpha^{*}$ for some leaf node $W_{\alpha^{*}}$. Then $\alpha \in \operatorname{rel}_{\mathcal{G}}(\mathbf{Y} \mid \boldsymbol{\alpha})$ by lemma 3.2.

LEMMA 0.5. There is a bijective correspondence between vertices in $\mathcal{F}(\mathbf{Y}, \boldsymbol{\alpha}, \mathcal{G})$, and $\operatorname{rel}_{\mathcal{G}}(\mathbf{Y} \mid \boldsymbol{\alpha})$. Specifically, the function that maps each vertex $W_{\alpha}$ in $\mathcal{F}(\mathbf{Y}, \boldsymbol{\alpha}, \mathcal{G})$ to $\alpha$ is bijective.

Proof. Fix a vertex $W_{\alpha}$ in $\mathcal{F}(\mathbf{Y}, \boldsymbol{\alpha}, \mathcal{G})$. Then $\alpha \in \operatorname{rel}_{\mathcal{G}}(\mathbf{Y} \mid \boldsymbol{\alpha})$ by lemma 0.4. That distinct vertices $W_{\alpha}$ and $Z_{\beta}$ in $\mathcal{F}(\mathbf{Y}, \boldsymbol{\alpha}, \mathcal{G})$ correspond to distinct relevant paths follows immediately by construction of $\mathcal{F}(\mathbf{Y}, \boldsymbol{\alpha}, \mathcal{G})$.

The converse follows by definition of (4), and the definition of relevant paths. 
Lemma 0.6. Consider a defined response to a path intervention $\pi$ corresponding to a vertex $W_{\alpha}$ in $\mathcal{F}(\mathbf{Y}, \boldsymbol{\alpha}, \mathcal{G})$. This response is equal to $W$ under no intervention if and only if for all paths in $\operatorname{rel}_{\mathcal{G}}(\mathbf{Y} \mid \boldsymbol{\alpha})$ that both contain $\alpha$ as a suffix subpath (or $W$ as a sink if $\alpha$ is the empty path), and have a prefix $\beta \in \boldsymbol{\alpha}, \pi$ sets $\beta$ to a natural value.

Proof. By definition of (4).

Lemma 0.7. If $\boldsymbol{\alpha}^{*} \subseteq \boldsymbol{\alpha}$, then any leaf node $W_{\alpha}$ in $\mathcal{F}(\mathbf{Y}, \boldsymbol{\alpha}, \mathcal{G})$ also exists in $\mathcal{F}\left(\mathbf{Y}, \boldsymbol{\alpha}^{*}, \mathcal{G}\right)$ (but is not necessarily a leaf).

Proof. Fix a leaf node $W_{\alpha}$ in $\mathcal{F}(\mathbf{Y}, \boldsymbol{\alpha}, \mathcal{G})$. By lemma $0.4, \alpha \in \operatorname{rel}_{\mathcal{G}}(\mathbf{Y}$ $\boldsymbol{\alpha})$. Since $\boldsymbol{\alpha}^{*} \subseteq \boldsymbol{\alpha}, \operatorname{rel}_{\mathcal{G}}(\mathbf{Y} \mid \boldsymbol{\alpha}) \subseteq \operatorname{rel}_{\mathcal{G}}\left(\mathbf{Y} \mid \boldsymbol{\alpha}^{*}\right)$ by lemma 3.3. Thus, $\alpha \in$ $\operatorname{rel}_{\mathcal{G}}\left(\mathbf{Y} \mid \boldsymbol{\alpha}^{*}\right)$. Then $W_{\alpha}$ is also a node in $\mathcal{F}\left(\mathbf{Y}, \boldsymbol{\alpha}^{*}, \mathcal{G}\right)$ by lemma 0.5 .

Lemma 3.1. If $\boldsymbol{\alpha}$ is proper, then for any edge $(W Y)_{\rightarrow}$, so is $\triangleleft_{(W Y) \rightarrow}(\boldsymbol{\alpha})$.

Proof. If $\triangleleft_{(W Y)_{\rightarrow}}(\boldsymbol{\alpha})$ is not proper, fix distinct witnessing paths $\alpha, \beta \in$ $\triangleleft_{(W Y) \rightarrow}(\boldsymbol{\alpha})$, where $\alpha$ is a prefix of $\beta$. If $\alpha, \beta \in \boldsymbol{\alpha}$, we have a contradiction. If $\beta \notin \boldsymbol{\alpha}$ but $\alpha \in \boldsymbol{\alpha}$, then there is a path $\beta^{*} \in \boldsymbol{\alpha}$ distinct from $\beta$, such that $\triangleleft_{(W Y) \rightarrow}\left(\beta^{*}\right)=\beta$. But by definition of the funnel operator, $\beta$ is a prefix of $\beta^{*}$. Since $\alpha$ is a prefix of $\beta$, it is also a prefix of $\beta^{*}$, and we have a contradiction. If $\beta \in \boldsymbol{\alpha}$ but $\alpha \notin \boldsymbol{\alpha}$, then $\alpha$ is a path of the form $(A \ldots W)_{\rightarrow}$. Since $\alpha$ is a prefix of $\beta$, then $\beta=(A \ldots W) \rightarrow$ (if $\beta$ were longer than this, it would have either been shortened or removed, in either case $\beta$ would not been a member of $\left.\triangleleft_{(W Y)_{\rightarrow}}(\boldsymbol{\alpha})\right)$. But we assumed $\alpha \neq \beta$. If $\beta \notin \boldsymbol{\alpha}$ and $\alpha \notin \boldsymbol{\alpha}$, then $\alpha$ must be of the form $(A \ldots W)_{\rightarrow}$. Since $\alpha$ is a prefix of $\beta$, either $\beta \notin \triangleleft_{(W Y) \rightarrow}(\boldsymbol{\alpha})$, or $\alpha=\beta$. Either way, we have a contradiction.

Lemma 3.2. If $\alpha \in \operatorname{rel}_{\mathcal{G}}(\mathbf{Y} \mid \boldsymbol{\alpha})$, then $\beta \in \operatorname{rel}_{\mathcal{G}}(\mathbf{Y} \mid \boldsymbol{\alpha})$ for any suffix subpath $\beta$ of $\alpha$.

Proof. Since $\operatorname{sink}_{\mathcal{G}}(\alpha) \in \mathbf{Y}$, and $\beta$ is a suffix subpath of $\alpha, \operatorname{sink}_{\mathcal{G}}(\alpha) \in \mathbf{Y}$. If no path in $\boldsymbol{\alpha}$ is in $\alpha$, no path in $\boldsymbol{\alpha}$ is in $\beta$. If a path in $\boldsymbol{\alpha}$ is a prefix subpath of $\alpha$ and no other element of $\alpha$ is in $\alpha$, then either $\beta=\alpha$ in which case the same is true for beta, or $\beta$ is a strict subpath of $\alpha$, in which case no element of $\boldsymbol{\alpha}$ is contained in $\beta$. In either case, $\beta \in \operatorname{rel}_{\mathcal{G}}(\mathbf{Y} \mid \boldsymbol{\alpha})$.

Lemma 3.3. If $\boldsymbol{\beta} \subseteq \boldsymbol{\alpha}$, then for any $\mathbf{Y}, \operatorname{rel}_{\mathcal{G}}(\mathbf{Y} \mid \boldsymbol{\alpha}) \subseteq \operatorname{rel}_{\mathcal{G}}(\mathbf{Y} \mid \boldsymbol{\beta})$.

Proof. Fix $\alpha \in \operatorname{rel}_{\mathcal{G}}(\mathbf{Y} \mid \boldsymbol{\alpha})$. If no element in $\boldsymbol{\alpha}$ is contained in $\alpha$, no element of $\boldsymbol{\beta}$ is contained in $\alpha$ since $\boldsymbol{\beta} \subseteq \boldsymbol{\alpha}$. If only a single element $\alpha^{*}$ in 
$\boldsymbol{\alpha}$ is contained in $\alpha$ as a prefix subpath, then either $\alpha^{*} \in \boldsymbol{\beta}$ or $\alpha^{*} \notin \boldsymbol{\beta}$. In the first case only a single element of $\boldsymbol{\beta}$ is contained in $\alpha$ and it is a prefix subpath. In the second case, no elements of $\boldsymbol{\beta}$ are contained in $\alpha$. In either case, $\alpha \in \operatorname{rel}_{\mathcal{G}}(\mathbf{Y} \mid \boldsymbol{\beta})$.

Lemma 3.4. For any $\mathbf{Y}$ and $\boldsymbol{\alpha}$ proper for $\mathbf{Y}, \operatorname{rel}_{\mathcal{G}}(\mathbf{Y} \mid \boldsymbol{\alpha})=\operatorname{rel}_{\mathcal{G}}\left(\mathbf{Y} \mid \boldsymbol{\alpha}_{\mathbf{Y}}\right)$, $\left(\boldsymbol{\alpha}_{\mathbf{Y}}\right)_{\mathbf{Y}}=\boldsymbol{\alpha}_{\mathbf{Y}}$, and in addition, for any $\mathfrak{a}_{\boldsymbol{\alpha}}, p\left(\mathbf{Y}\left(\mathfrak{a}_{\boldsymbol{\alpha}}\right)\right)=p\left(\mathbf{Y}\left(\mathfrak{a}_{\boldsymbol{\alpha}_{\mathbf{Y}}}\right)\right)$.

Proof. That $\operatorname{rel}_{\mathcal{G}}(\mathbf{Y} \mid \boldsymbol{\alpha}) \subseteq \operatorname{rel}_{\mathcal{G}}\left(\mathbf{Y} \mid \boldsymbol{\alpha}_{\mathbf{Y}}\right)$ follows from the fact that $\boldsymbol{\alpha}_{\mathbf{Y}} \subseteq \boldsymbol{\alpha}$, and lemma 3.3.

Fix $\alpha \in \operatorname{rel}_{\mathcal{G}}\left(\mathbf{Y} \mid \boldsymbol{\alpha}_{\mathbf{Y}}\right)$. If $\alpha$ does not contain subpaths in $\boldsymbol{\alpha} \backslash \boldsymbol{\alpha}_{\mathbf{Y}}$, then $\alpha \in \operatorname{rel}_{\mathcal{G}}(\mathbf{Y} \mid \boldsymbol{\alpha})$. Otherwise, assume $\alpha$ contains subpaths in $\boldsymbol{\alpha} \backslash \boldsymbol{\alpha}_{\mathbf{Y}}$. Choose such a subpath with a source closest to the $\operatorname{sink}$ of $\alpha$, call it $\beta$. Then the suffix subpath $\alpha^{\dagger}$ of $\alpha$ which contains $\beta$ as a prefix either does or does not contain other paths in $\boldsymbol{\alpha}_{\mathbf{Y}}$. If it does, we have a contradiction with our assumption that $\alpha \in \operatorname{rel}_{\mathcal{G}}\left(\mathbf{Y} \mid \boldsymbol{\alpha}_{\mathbf{Y}}\right)$. If it does not, then since by construction $\alpha^{\dagger}$ does not contain any elements in $\boldsymbol{\alpha} \backslash \boldsymbol{\alpha}_{\mathbf{Y}}$ other than $\beta, \alpha^{\dagger}$ does not contain any paths in $\boldsymbol{\alpha}$ other than $\beta$. Thus $\alpha^{\dagger} \in \operatorname{rel}_{\mathcal{G}}(\mathbf{Y} \mid \boldsymbol{\alpha})$, and therefore $\beta \in \boldsymbol{\alpha}_{\mathbf{Y}}$. But this contradicts our assumption that $\beta \in \boldsymbol{\alpha} \backslash \boldsymbol{\alpha}_{\mathbf{Y}}$. Thus $\operatorname{rel}_{\mathcal{G}}\left(\mathbf{Y} \mid \boldsymbol{\alpha}_{\mathbf{Y}}\right) \subseteq$ $\operatorname{rel}_{\mathcal{G}}(\mathbf{Y} \mid \boldsymbol{\alpha})$.

By definition, $\left(\boldsymbol{\alpha}_{\mathbf{Y}}\right)_{\mathbf{Y}} \subseteq \boldsymbol{\alpha}_{\mathbf{Y}}$. Fix $\alpha \in \boldsymbol{\alpha}_{\mathbf{Y}}$. Then there is $\beta \in \operatorname{rel}_{\mathcal{G}}(\mathbf{Y} \mid \boldsymbol{\alpha})$ with $\alpha$ as a prefix. But by above argument, $\beta \in \operatorname{rel}_{\mathcal{G}}\left(\mathbf{Y} \mid \boldsymbol{\alpha}_{\mathbf{Y}}\right)$. Since $\beta$ does not contain elements of $\boldsymbol{\alpha}$ except $\alpha$ it also does not contain any elements of $\boldsymbol{\alpha}_{\mathbf{Y}}$ except $\alpha$. Thus $\alpha \in\left(\boldsymbol{\alpha}_{\mathbf{Y}}\right)_{\mathbf{Y}}$.

To see that $p\left(\mathbf{Y}\left(\mathfrak{a}_{\boldsymbol{\alpha}}\right)\right)=p\left(\mathbf{Y}\left(\mathfrak{a}_{\boldsymbol{\alpha}_{\mathbf{Y}}}\right)\right)$, note that $\operatorname{since} \operatorname{rel}_{\mathcal{G}}(\mathbf{Y} \mid \boldsymbol{\alpha})=$ $\operatorname{rel}_{\mathcal{G}}\left(\mathbf{Y} \mid \boldsymbol{\alpha}_{\mathbf{Y}}\right), \mathcal{F}(\mathbf{Y}, \boldsymbol{\alpha}, \mathcal{G})$ and $\mathcal{F}\left(\mathbf{Y}, \boldsymbol{\alpha}_{\mathbf{Y}}, \mathcal{G}\right)$ are the same by lemma 0.5 , and since $\boldsymbol{\alpha}_{\mathbf{Y}}=\left(\boldsymbol{\alpha}_{\mathbf{Y}}\right)_{\mathbf{Y}}$, all leaf nodes in $\mathcal{F}(\mathbf{Y}, \boldsymbol{\alpha}, \mathcal{G})$ and $\mathcal{F}\left(\mathbf{Y}, \boldsymbol{\alpha}_{\mathbf{Y}}, \mathcal{G}\right)$ are assigned the same values by $\pi_{\mathfrak{a}_{\alpha}}$ and $\pi_{\mathfrak{a}_{\alpha_{\mathbf{Y}}}}$, respectively.

Lemma 3.5. Let $\pi_{\mathfrak{a}_{\alpha}}$ be a path intervention natural for $\mathbf{Y}$, and $\boldsymbol{\alpha}^{*} \subseteq$ $\boldsymbol{\alpha}$ is those paths which are assigned constant values. Then $p\left(\mathbf{Y}\left(\mathfrak{a}_{\boldsymbol{\alpha}}\right)\right)=$ $p\left(\mathbf{Y}\left(\mathfrak{a}_{\boldsymbol{\alpha}^{*}}\right)\right)$.

Proof. Fix a leaf node $W_{\alpha}$ in $\mathcal{F}(\mathbf{Y}, \boldsymbol{\alpha}, \mathcal{G})$. By lemma $0.7, W_{\alpha}$ also exists in $\mathcal{F}\left(\mathbf{Y}, \boldsymbol{\alpha}^{*}, \mathcal{G}\right)$ (but is not necessarily a leaf).

If $W_{\alpha}$ is also a leaf node in $\mathcal{F}\left(\mathbf{Y}, \boldsymbol{\alpha}^{*}, \mathcal{G}\right)$, then either both corresponding defined responses are equal to the same constant assigned to some element of $\boldsymbol{\alpha}^{*}$, or to a natural value of a variable corresponding to a node in $\mathcal{G}$ with no parents. In either case, the responses are the same.

If $W_{\alpha}$ is not a leaf node in $\mathcal{F}\left(\mathbf{Y}, \boldsymbol{\alpha}^{*}, \mathcal{G}\right)$, by definition of $\boldsymbol{\alpha}^{*}$ this implies $\alpha$ is an element of $\operatorname{rel}_{\mathcal{G}}(\mathbf{Y} \mid \boldsymbol{\alpha})$, and has a prefix in $\boldsymbol{\alpha} \backslash \boldsymbol{\alpha}^{*}$. Assume the defined 
response to $\pi_{\mathfrak{a}_{\alpha^{*}}}$ corresponding to $W_{\alpha}$ is not a natural response. lemma 0.6 then implies the existence of a path $\alpha^{\dagger}$ in $\mathcal{G}$ which contains a prefix subpath $\beta$ in $\boldsymbol{\alpha}^{*}$, and a suffix subpath $\alpha$. But $\alpha$ contains a prefix in $\boldsymbol{\alpha} \backslash \boldsymbol{\alpha}^{*}$ which implies $\pi$ is not natural for $\mathbf{Y}$ as we assumed. This is a contradiction.

Since all leaf nodes in $\mathcal{F}\left(\mathbf{Y}, \boldsymbol{\alpha}^{*}, \mathcal{G}\right)$ agree on their values with corresponding nodes in $\mathcal{F}(\mathbf{Y}, \boldsymbol{\alpha}, \mathcal{G})$, our conclusion follows.

Lemma 3.6. If $\pi_{\mathfrak{a}_{\boldsymbol{\alpha}}}$ is not natural for $\mathbf{Y}$ in $\mathcal{G}$, then $p\left(\mathbf{Y}\left(\mathfrak{a}_{\boldsymbol{\alpha}}\right)\right)$ is not identified under the $M W M$ for $\mathcal{G}$.

Proof. If $\pi$ is not natural, fix $\gamma \in \operatorname{rel}_{\mathcal{G}}\left(\mathbf{Y} \mid \boldsymbol{\alpha}^{*}\right)$ with a prefix subpath $\alpha$ in $\boldsymbol{\alpha}^{*}$, such that $\gamma$ contains a subpath in $\boldsymbol{\alpha} \backslash \boldsymbol{\alpha}^{*}$. In fact, we shall pick $\beta \in \boldsymbol{\alpha} \backslash \boldsymbol{\alpha}^{*}$ in $\gamma$ with the property that no other element in $\boldsymbol{\alpha} \backslash \boldsymbol{\alpha}^{*}$ contained in $\gamma$ has a source node between $\operatorname{sog}_{\mathcal{G}}(\beta)$ and $\operatorname{so}_{\mathcal{G}}(\alpha)$. Such $\beta$ exists by assumption, and is unique since $\boldsymbol{\alpha}$ is a proper set.

Since $\boldsymbol{\alpha}$ was assumed to be live for $\mathbf{Y}$, there exist paths $\alpha^{*}, \beta^{*} \in \operatorname{rel}_{\mathcal{G}}(\mathbf{Y}$ $\boldsymbol{\alpha})$ containing $\alpha, \beta$ as prefix subpaths, respectively. We will consider an edge subgraph $\mathcal{G}^{*}$ of $\mathcal{G}$ containing only edges which appear in $\alpha^{*}, \beta^{*}$, and a subpath of $\gamma$ containing $\alpha, \beta$, and a subpath between them (if any). Since $\alpha^{*}, \beta^{*} \in \operatorname{rel}_{\mathcal{G}}(\mathbf{Y} \mid \boldsymbol{\alpha})$, no elements of $\boldsymbol{\alpha}$ other than $\alpha, \beta$ appear in those paths. Since $\gamma \in \operatorname{rel}_{\mathcal{G}}\left(\mathbf{Y} \mid \boldsymbol{\alpha}^{*}\right)$, no element of $\boldsymbol{\alpha}^{*}$ appears in $\gamma$ other than $\alpha$. By assumption on $\beta$, no element of $\boldsymbol{\alpha} \backslash \boldsymbol{\alpha}^{*}$ other than $\beta$ appears in the subpath of $\gamma$ with edges which appear in $\mathcal{G}^{*}$. Thus $\alpha, \beta$ are the only elements of $\boldsymbol{\alpha}$ that appear in $\mathcal{G}^{*}$.

Since $\alpha^{*} \in \operatorname{rel}_{\mathcal{G}}(\mathbf{Y} \mid \boldsymbol{\alpha}), \beta$ is not a subpath of $\alpha^{*}$. This implies $\gamma$ is not a subpath of $\alpha^{*}$, but both share $\alpha$ as a prefix subpath by construction. Let $B$ be the second node in $\alpha$ after the source. By lemma 5.8, $p\left(B, B\left(\mathfrak{a}_{\alpha}\right)\right)$ is not identified under the MWM for $\mathcal{G}^{*}$. This then implies by an argument in theorem 5.2 that $p\left(\operatorname{sink}_{\mathcal{G}}\left(\left\{\alpha^{*}, \beta^{*}\right\}\right)\left(\mathfrak{a}_{\{\alpha, \beta\}}\right)\right)$ is not identified under the MWM for $\mathcal{G}^{*}$, which in turn implies the overall response is not identified under the MWM for $\mathcal{G}$ by lemma 5.5.

Proofs for Section 4 .

Lemma 4.1. $\boldsymbol{\alpha}_{\mathbf{A}, \mathbf{Y}, \mathcal{G}}$ is always proper.

Proof. If not, there exists $\alpha, \beta \in \boldsymbol{\alpha}_{\mathbf{A}, \mathbf{Y}, \mathcal{G}}$, where $\alpha$ is a prefix subpath of $\beta$. The definition of $\boldsymbol{\alpha}_{\mathbf{A}, \mathbf{Y}, \mathcal{G}}$ implies $\operatorname{sink}_{\mathcal{G}}(\alpha) \in \mathbf{A} \cup \mathbf{Y}$, and $\operatorname{since} \operatorname{sink}_{\mathcal{G}}(\alpha) \neq$ $\operatorname{sink}_{\mathcal{G}}(\beta), \beta$ intersects $\mathbf{A} \cup \mathbf{Y}$ at a node that is neither source nor sink. This contradicts our assumption. 
Lemma 4.2. If there exists $A \in \mathbf{A}$ such that $A\left(\mathfrak{a}_{\boldsymbol{\alpha}_{\mathbf{A}, \mathbf{Y}, \mathcal{G}}}\right) \neq A, p(\mathbf{Y}(\mathbf{a}), \mathbf{A})$ is not identified under the $M W M$ for $\mathcal{G}$. If there does not exist such an $A$, $p(\mathbf{Y}(\mathbf{a}), \mathbf{A})=p\left(\{\mathbf{Y} \cup \mathbf{A}\}\left(\mathfrak{a}_{\boldsymbol{\alpha}_{\mathbf{A}, \mathbf{Y}, \mathcal{G}}}\right)\right)$.

Proof. By lemma 0.6, if there exists $A \in \mathbf{A}$ such that $A\left(\mathfrak{a}_{\boldsymbol{\alpha}_{\mathbf{A}, \mathbf{Y}, \mathcal{G}}}\right) \neq A$, there must exist $\alpha \in \operatorname{rel}_{\mathcal{G}}\left(\mathbf{Y} \cup \mathbf{A} \mid \boldsymbol{\alpha}_{\mathbf{A}, \mathbf{Y}, \mathcal{G}}\right)$ with a prefix subpath $\beta \in \boldsymbol{\alpha}_{\mathbf{A}, \mathbf{Y}, \mathcal{G}}$ set to a constant, such that $\operatorname{sink}_{\mathcal{G}}(\alpha)=A$. Let $\operatorname{sink}_{\mathcal{G}}(\beta)=Y \in \mathbf{Y}$.

Consider the edge subgraph $\mathcal{G}^{*}$ of $\mathcal{G}$ which only contains edges in $\alpha$. Since $\alpha \in \operatorname{rel}_{\mathcal{G}}\left(\mathbf{Y} \cup \mathbf{A} \mid \boldsymbol{\alpha}_{\mathbf{A}, \mathbf{Y}, \mathcal{G}}\right), \beta$ is the only element of $\boldsymbol{\alpha}_{\mathbf{A}, \mathbf{Y}, \mathcal{G}}$ in $\mathcal{G}^{*}$. Let $B$ be the second node after the source in $\alpha$. By lemma $5.8, p\left(B, B\left(\mathfrak{a}_{\beta}\right)\right)$ is not identified under the MWM for $\mathcal{G}^{*}$. This implies, using the path argument

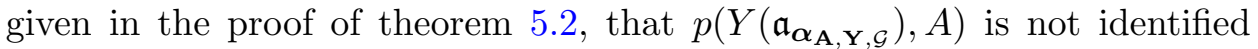
under the MWM of $\mathcal{G}^{*}$. By lemma 5.1, $p\left(Y\left(\mathfrak{a}_{\left.\boldsymbol{\alpha}_{\mathbf{A}, \mathbf{Y}, \mathcal{G}}\right)}\right), A\right)=p(Y(\mathbf{a}), A)$ in $\mathcal{G}^{*}$. Since $\alpha \in \operatorname{rel}_{\mathcal{G}}\left(\mathbf{Y} \cup \mathbf{A} \mid \boldsymbol{\alpha}_{\mathbf{A}, \mathbf{Y}, \mathcal{G}}\right), \beta$ is the only element of $\boldsymbol{\alpha}_{\mathbf{A}, \mathbf{Y}, \mathcal{G}}$ in $\mathcal{G}^{*}$. This implies $p(\mathbf{Y}(\mathbf{a}), \mathbf{A})$ is not identified under the MWM for $\mathcal{G}$ by lemma 5.5.

Otherwise, $\mathbf{A}\left(\mathfrak{a}_{\boldsymbol{\alpha}_{\mathbf{A}, \mathbf{Y}, \mathcal{G}}}\right)=\mathbf{A}$ by assumption, and $\mathbf{Y}\left(\mathfrak{a}_{\boldsymbol{\alpha}_{\mathbf{A}, \mathbf{Y}, \mathcal{G}}}\right)=\mathbf{Y}(\mathbf{a})$ by lemma 5.1.

Proofs for section 5.

Lemma 5.1. Let $\mathbf{A}, \mathbf{Y}$ be disjoint vertex sets in a $D A G \mathcal{G}$, and $\mathbf{a}$ a value assignment to $\mathbf{A}$. Let $\pi_{\mathfrak{a}_{\boldsymbol{\alpha}}, \mathbf{Y}, \mathcal{G}}^{\mathbf{a}}$ assign each $\alpha \in \boldsymbol{\alpha}_{\mathbf{A}, \mathbf{Y}, \mathcal{G}}$ to $\mathbf{a}_{\mathbf{s o}_{\mathcal{G}}(\alpha)}$. Then $p\left(\mathbf{Y}\left(\mathfrak{a}_{\boldsymbol{\alpha} \mathbf{A}, \mathbf{Y}, \mathcal{G}}\right)\right)=p(\mathbf{Y}(\mathbf{a}))$.

Proof. By definition of recursive substitution for node interventions, the defined response for each $Y \in \mathbf{Y}$ to a node intervention setting $\mathbf{A}$ to $\mathbf{a}$ is an expression tree with paths corresponding to directed paths from $\mathbf{A}$ to $Y$ that do not intersect elements of $\mathbf{A}$ other than at source, and directed paths from parent-less nodes in $\mathcal{G}$ to $Y$ that do not intersect $\mathbf{A}$. But these paths are precisely paths in $\operatorname{rel}_{\mathcal{G}}\left(Y \mid \boldsymbol{\alpha}_{\mathbf{A}, \mathbf{Y}, \mathcal{G}}\right)$ which either have prefixes in $\boldsymbol{\alpha}_{\mathbf{A}, \mathbf{Y}, \mathcal{G}}$ or parent-less source nodes, which are paths that correspond to paths from leaves to $Y$ in $\mathcal{F}\left(\mathbf{Y}, \boldsymbol{\alpha}_{\mathbf{A}, \mathbf{Y}, \mathcal{G}}, \mathcal{G}\right)$. That the defined responses are the same follows by the way $\pi^{\mathbf{a}}$ was defined.

Lemma 5.2. Let $\mathbf{Y}$ be a vertex set in a DAG $\mathcal{G}$, and $\boldsymbol{\alpha}$ a set of edges, with $\mathfrak{a}_{\boldsymbol{\alpha}}$ an assignment to $\boldsymbol{\alpha}$. Let $\mathbf{A}=\operatorname{so}_{\mathcal{G}}(\boldsymbol{\alpha})$, and $\boldsymbol{\alpha}_{\mathbf{Y}, \mathcal{G}}$ be a subset of $\boldsymbol{\alpha}_{\mathbf{A}, \mathbf{Y}, \mathcal{G}}$ consisting of paths with an edge prefix in $\boldsymbol{\alpha}$. Let $\pi_{\mathfrak{a}_{\mathbf{\alpha}, \mathcal{G}}}^{\boldsymbol{\alpha}}$ assign each $\alpha \in \boldsymbol{\alpha}_{\mathbf{Y}, \mathcal{G}}$ to the value assigned to the edge prefix of $\alpha$ by $\mathfrak{a}_{\boldsymbol{\alpha}}$. Then $p\left(\mathbf{Y}\left(\mathfrak{a}_{\boldsymbol{\alpha} \mathbf{Y}, \mathcal{G}}\right)\right)=$ $p\left(\mathbf{Y}\left(\mathfrak{a}_{\boldsymbol{\alpha}}\right)\right)$.

Proof. By definition of recursive substitution for edge interventions, the defined response for each $Y \in \mathbf{Y}$ to an edge intervention setting $\boldsymbol{\alpha}$ to $\mathfrak{a}_{\boldsymbol{\alpha}}$ is an 
expression tree with paths corresponding to elements of $\operatorname{rel}_{\mathcal{G}}(\mathbf{Y} \mid \boldsymbol{\alpha})$ which either contain an edge prefix in $\boldsymbol{\alpha}$ or have a parent-less source in $\mathcal{G}$. But these paths are precisely paths in $\operatorname{rel}_{\mathcal{G}}\left(Y \mid \boldsymbol{\alpha}_{\mathbf{Y}, \mathcal{G}}\right)$ which either have prefixes in $\boldsymbol{\alpha}_{\mathbf{Y}, \mathcal{G}}$ or parent-less source nodes, which are paths that correspond to paths from leaves to $Y$ in $\mathcal{F}\left(\mathbf{Y}, \boldsymbol{\alpha}_{\mathbf{Y}, \mathcal{G}}, \mathcal{G}\right)$. That the defined responses are the same follows by the way $\pi^{\alpha}$ was defined.

Consider the following two sets of random variables $\mathbf{V}_{1}$ and $\mathbf{V}_{2}$ associated with vertices $\mathbf{V}=\left\{A, B, C, U_{B}, U_{C}\right\}$, defined as follows:

$\begin{array}{llllll} & A & U_{B} & U_{C} & B & C \\ \mathbf{V}_{1} & \operatorname{unif}(\{0,1\}) & \operatorname{unif}(\{0,1\}) & \operatorname{unif}(\{0,1\}) & A \oplus U_{B} & A \oplus U_{C} \\ \mathbf{V}_{2} & \operatorname{unif}(\{0,1\}) & \operatorname{unif}(\{0,1\}) & \operatorname{unif}(\{0,1\}) & U_{B}^{A} \cdot U_{C}^{(1-A)} & U_{B}^{(1-A)} \cdot U_{C}^{A}\end{array}$

where $\oplus$ denotes addition modulo 2 , and unif $(\mathbf{W})$ denotes a uniform distribution over values in a set $\mathbf{W}$.

Let $\mathcal{G}_{2}$ be the DAG shown in Fig. 3 (b). We define two causal structures $c_{1}\left(\{A, B, C\}, \mathcal{G}_{2}\right)$ and $c_{2}\left(\{A, B, C\}, \mathcal{G}_{2}\right)$ by first interpreting the functions defining the random variables as imperative computer programs, where variable replacement is possible (the so called structural equations), and second by considering the joint distribution over the resulting potential outcomes induced by the distributions over $U_{B}$ and $U_{C}$. In other words, for any values $b, c$ of $B$ and $C$, we have $A=A(b)=A(c)=A(b, c), B(a)=B(a, c)=a \oplus U_{B}$ in $c_{1}$, and $B(a)=B(a, c)=U_{B}^{a} \cdot U_{C}^{(1-a)}$ in $c_{2}$, with $C(a), C(a, b)$ defined similarly by symmetry. Though only counterfactuals for $\{A, B, C\}$ were defined, in fact $U_{B}$ and $U_{C}$ were also involved. If we include these variables as vertices, then the graph in Fig. 3 (c) represents the recipe for $c_{1}$, while the graph in Fig. 3 (d) represents the recipe for $c_{2}$. These types of recipes is how structural equation models are generally defined (see [2] for many examples).

Lemma 0.8. $\quad c_{1}\left(\{A, B, C\}, \mathcal{G}_{2}\right)$ and $c_{2}\left(\{A, B, C\}, \mathcal{G}_{2}\right)$ are in the $S W M$ associated with the DAG $\mathcal{G}_{2}$ in Fig. $3(b)$.

Proof. We must show that for all $a \in \mathfrak{X}_{A}$, the variables in the set $\{A, B(a), C(a)\}$ are mutually independent. In $c_{1}\left(\{A, B, C\}, \mathcal{G}_{2}\right)$ this follows from the fact that $A, U_{B}$ and $U_{C}$ are mutually independent, while in $c_{2}\left(\{A, B, C\}, \mathcal{G}_{2}\right)$ we have $B(1)=U_{B}, C(1)=U_{C}$. Thus $B(0)=U_{C}, C(0)=$ $U_{B}$, and mutual independence follows from the fact that $A, U_{B}$ and $U_{C}$ are mutually independent.

For a SWM for a DAG $\mathcal{G}$ with a vertex set $\mathbf{V}$, define $p(\mathbf{V}(*)) \equiv\{p(\mathbf{V}(\mathbf{a})) \mid$ $\left.\mathbf{A} \subseteq \mathbf{V}, \mathbf{a} \in \mathfrak{X}_{\mathbf{A}}\right\}$. 
Lemma 0.9. The structures $c_{1}(\{A, B, C\})$ and $c_{2}(\{A, B, C\})$ agree on the set $p(\{A, B, C\}(*))$.

Proof. By definition of recursive substitution for node interventions, it suffices to show $c_{1}$ and $c_{2}$ agree on $p(A, B, C)$ and $p(\{A, B, C\}(a))$ for all $a \in \mathfrak{X}_{A}$. By construction of $c_{1}$ and $c_{2}, p(A, B, C)=p(\{A, B, C\}(a)=$ unif $\left(\{0,1\}^{3}\right)$ in both causal structures for all $a \in \mathfrak{X}_{A}$.

Lemma 5.4. Responses $p\left(\{B, C\}\left((a B)_{\rightarrow},\left(a^{\prime} C\right)_{\rightarrow}\right)\right), p\left(\{B, C\}\left((a B)_{\rightarrow}\right)\right)$, and $p\left(\{B, C\}\left((a C)_{\rightarrow}\right)\right)$, are not identifiable from $p(A, B, C)$ under the $S W M$ for Fig. $3(b)$.

Proof. By definition, $p\left(\{B, C\}\left((a B)_{\rightarrow},\left(a^{\prime} C\right)_{\rightarrow}\right)\right)=p\left(U_{B}, 1-U_{C}\right)=$ unif $\left(\{0,1\}^{2}\right)$ in $c_{1}$, while in $c_{2}, p\left(\{B, C\}\left((a B)_{\rightarrow},\left(a^{\prime} C\right)_{\rightarrow}\right)\right)=p\left(U_{C}, U_{C}\right)=$ $\operatorname{unif}(\{00,11\})$.

Similarly, $p\left(\{B, C\}\left((a B)_{\rightarrow}\right)\right)=p\left(U_{B}, A \oplus U_{C}\right)=\operatorname{unif}\left(\{0,1\}^{2}\right)$ in $c_{1}$, while $p\left(\{B, C\}\left((a B)_{\rightarrow}\right)\right)=p\left(U_{C}, U_{B}^{(1-A)} \cdot U_{C}^{A}\right)$. But

$$
\begin{aligned}
p\left(U_{C}, U_{B}^{(1-A)} \cdot U_{C}^{A}\right) & =p\left(U_{C}, U_{C} \mid A=1\right) p(A=1)+p\left(U_{C}, U_{B} \mid A=0\right) p(A=0) \\
& =\operatorname{unif}(\{00,11\}) \cdot 0.5+\operatorname{unif}\left(\{0,1\}^{2}\right) \cdot 0.5,
\end{aligned}
$$

which is not a uniform distribution. By symmetry, the same argument applies to $p\left(\{B, C\}\left((a C)_{\rightarrow}\right)\right.$.

Lemma 5.5. Let $\mathcal{G}$ be a $D A G, \mathbf{Y}, \mathbf{A}$ disjoint sets of vertices in $\mathcal{G}, \boldsymbol{\alpha}$ a set of paths live for $\mathbf{Y}$. Let $\mathcal{G}^{*}$ be any edge supergraph of $\mathcal{G}, \mathbf{Y}^{*}$ any superset of $\mathbf{Y}$ in $\mathcal{G}^{*}, \boldsymbol{\alpha}^{*}$ a superset of $\boldsymbol{\alpha}$ in $\mathcal{G}^{*}$ live and proper for $\mathbf{Y}^{*}$, such that every path in $\boldsymbol{\alpha}^{*} \backslash \boldsymbol{\alpha}$ does not exist in $\mathcal{G}$. Finally, let $\pi_{\mathfrak{a}_{\alpha^{*}}}$ be a path intervention. If $p\left(\mathbf{Y}\left(\mathfrak{a}_{\boldsymbol{\alpha}}\right), \mathbf{A}\right)$ is not identified under the $M W M(S W M)$ for $\mathcal{G}$, then $p\left(\mathbf{Y}^{*}\left(\mathfrak{a}_{\boldsymbol{\alpha}^{*}}\right), \mathbf{A}\right)$ and $p\left(\mathbf{Y}\left(\mathfrak{a}_{\boldsymbol{\alpha}^{*}}\right), \mathbf{A} \cup \mathbf{Y}^{*} \backslash \mathbf{Y}\right)$ are not identified under the $M W M(S W M)$ for $\mathcal{G}^{*}$.

Proof. Any causal structure in the MWM (SWM) for $\mathcal{G}$ is also in the MWM (SWM) for $\mathcal{G}^{*}$. Thus the two causal structures that witness nonidentification of $p\left(\mathbf{Y}\left(\mathfrak{a}_{\boldsymbol{\alpha}}\right), \mathbf{A}\right)$ in $\mathcal{G}$ will also witness non-identification of $p\left(\mathbf{Y}\left(\mathfrak{a}_{\boldsymbol{\alpha}}\right), \mathbf{A}\right)$ in $\mathcal{G}^{*}$. Moreover, since no path in $\boldsymbol{\alpha}^{*} \backslash \boldsymbol{\alpha}$ exists in $\mathcal{G}$, in the two structures in question (though not in all structures in the MWM or SWM for $\left.\mathcal{G}^{*}\right), p\left(\mathbf{Y}\left(\mathfrak{a}_{\boldsymbol{\alpha}}\right), \mathbf{A}\right)=p\left(\mathbf{Y}\left(\mathfrak{a}_{\boldsymbol{\alpha}^{*}}\right), \mathbf{A}\right)$. Thus, $p\left(\mathbf{Y}\left(\mathfrak{a}_{\boldsymbol{\alpha}^{*}}\right), \mathbf{A}\right)$ is also not identifiable under the MWM (SWM) in $\mathcal{G}^{*}$.

If $p\left(\mathbf{Y}\left(\mathfrak{a}_{\boldsymbol{\alpha}^{*}}\right), \mathbf{A} \cup \mathbf{Y}^{*} \backslash \mathbf{Y}\right)$ or $p\left(\mathbf{Y}^{*}\left(\mathfrak{a}_{\boldsymbol{\alpha}^{*}}\right), \mathbf{A}\right)$ were identified under the MWM $(\mathrm{SWM})$ for $\mathcal{G}^{*}$, then we could obtain $p\left(\mathbf{Y}\left(\mathfrak{a}_{\boldsymbol{\alpha}^{*}}\right), \mathbf{A}\right)$ by marginalization. But we showed above $p\left(\mathbf{Y}\left(\mathfrak{a}_{\boldsymbol{\alpha}^{*}}\right), \mathbf{A}\right)$ is not identified. 
(a)

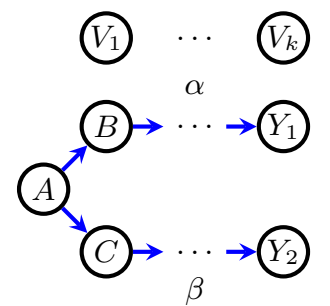

(b)

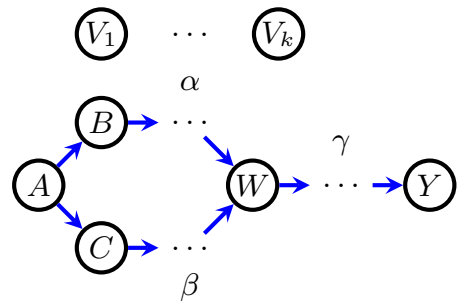

FIG 8. A schematic representation of graphs used in the proof of theorem 5.1.

Theorem 5.1. Consider a DAG $\mathcal{G}$ with vertices $\mathbf{V}$, and a set of edges $\boldsymbol{\alpha}$ live for $\mathbf{Y}$. Then $p\left(\mathbf{Y}\left(\mathfrak{a}_{\boldsymbol{\alpha}}\right)\right)$ is identifiable from $p(\mathbf{V})$ under the $S W M$ for $\mathcal{G}$ if and only if $\eta_{\mathfrak{a}_{\boldsymbol{\alpha}}}$ is node consistent. Moreover, if $p\left(\mathbf{Y}\left(\mathfrak{a}_{\boldsymbol{\alpha}}\right)\right)$ is identifiable, it is equal to the appropriate marginal of the extended $g$-formula (2) for $p\left(\mathbf{Y}\left(\mathbf{a}_{\boldsymbol{\alpha}}\right)\right)$, the response to the induced node intervention.

PROOF. If $\eta_{\mathfrak{a}_{\alpha}}$ is node consistent, then identification follows by lemma 5.3 and corollary 5.1 .

If $\eta_{\mathfrak{a}_{\alpha}}$ is node inconsistent, we will show non-identification of $p\left(\mathbf{Y}\left(\mathfrak{a}_{\boldsymbol{\alpha}}\right)\right)$ first in a simple edge subgraph $\mathcal{G}^{*}$ of $\mathcal{G}$, and then extend the result to $\mathcal{G}$ via lemma 5.5.

Pick edges $(A B) \rightarrow$ and $(A C)_{\rightarrow}$ with a common source and live for $\mathbf{Y}$, which are either assigned different values by $\eta_{\mathfrak{a}_{\boldsymbol{\alpha}}}$, or only one is in $\boldsymbol{\alpha}$. We will show non-identification with respect to the SWM model of certain edge subgraphs of $\mathcal{G}$ first.

We have two cases. In both cases we have a directed path $\alpha$ which starts with $B$ and does not intersect $\boldsymbol{\alpha}$, and a directed path $\beta$ which starts with $C$ and does not intersect $\boldsymbol{\alpha}$.

The first case is if there are distinct $Y_{1}, Y_{2} \in \mathbf{Y}$ such that $B \in \operatorname{an}_{\mathcal{G}}\left(Y_{1}\right)$ along a path $\alpha=\left(B \ldots Y_{1}\right)_{\rightarrow}$, and $C \in \operatorname{an}_{\mathcal{G}}\left(Y_{2}\right)$ along a path $\beta=\left(C \ldots Y_{2}\right)_{\rightarrow}$. The second case is if there is a single $Y \in \mathbf{Y}$ such that $B \in \operatorname{an}_{\mathcal{G}}(Y)$ along a path $\alpha=(B \ldots W)_{\rightarrow}$ appended with another path $\gamma=(W \ldots Y)_{\rightarrow}$, and $C \in \operatorname{an}_{\mathcal{G}}(Y)$ along a path $\beta=(C \ldots W)_{\rightarrow}$ appended with $\gamma=(W \ldots Y) \rightarrow$, where $\gamma$ also does not intersect any edge in $\boldsymbol{\alpha}$.

We are going to show non-identification with respect to two subgraphs of $\mathcal{G}$ corresponding to these cases, shown schematically in Fig. 8, with the graph in (a) corresponding to case 1, and the graph in (b) corresponding to case 2. We label vertices in $\mathbf{V}$ not on the paths from $A$ to $\left\{Y_{1}, Y_{2}\right\}$ for case 1 or not on the paths from $A$ to $Y$ for case 2 as $V_{1}, \ldots V_{k}$.

We consider binary state spaces, extensions to larger state spaces are 
not difficult. Our plan is to extend the causal structures $c_{1}$ and $c_{2}$ for the graph in Fig. 3 (b) to the graphs in Fig. 8 (a) (in which case we call them $c_{1}^{a}, c_{2}^{a}$ ) and (b) (in which case we call them $c_{1}^{b}, c_{1}^{b}$ ) in such a way that these extended causal structures are in the SWM with respect to the appropriate graph, agree on $\left\{p\left(\mathbf{V}(\mathbf{a}) \mid \mathbf{a} \in \mathfrak{X}_{\mathbf{A}}, \mathbf{A} \subseteq \mathbf{V}\right\}\right.$, but disagree on $p\left(\left\{Y_{1}, Y_{2}\right\}\left(\mathfrak{a}_{\left\{(A B)_{\rightarrow},(A C)_{\rightarrow}\right\}}\right)\right)$ for case 1 , and $p\left(Y\left(\mathfrak{a}_{\left\{(A B)_{\rightarrow,}(A C)_{\rightarrow}\right\}}\right)\right)$ for case 2 .

In all four causal structures $c_{1}^{a}, c_{2}^{a}, c_{1}^{b}, c_{2}^{b}$, and for every $V_{i} \in\left\{V_{1}, \ldots V_{k}\right\}$, every $\mathbf{A} \subseteq \mathbf{V}$, we let $V_{i}=V_{i}(\mathbf{a})=\operatorname{unif}(\{0,1\})$, for all $\mathbf{a} \in \mathfrak{X}_{\mathbf{A}}$.

Now consider any edge $(D E)_{\rightarrow}$ in $\alpha$ or $\beta$ in Fig. 8 (a), or in $\alpha$ or $\beta$ in Fig. 8 (b) such that $E \neq W$, or in $\gamma$ in Fig. 8 (b). We define $p(E(d))$ for $d \in \mathfrak{X}_{D}=\{0,1\}$ in such a way that whenever $p_{1}^{*}(D) \neq p_{2}^{*}(D)$,

$$
\left(\sum_{d} p(E(d)) p_{1}^{*}(D=d)\right) \neq\left(\sum_{d} p(E(d)) p_{2}^{*}(D=d)\right)
$$

In other words, we define $p(E(d))$ in such a way that

$$
f_{(D E)_{\rightarrow}}\left(p^{*}(D)\right) \mapsto p^{*}(E):\left(\sum_{d} p(E(d)) p^{*}(D=d)\right)
$$

is a one to one linear map from distributions over $D$ to distributions over $E$. Such mappings clearly exist: it suffices to consider an invertible 2 by 2 matrix with entries between 0 and 1 , where all columns sum to 1 . We allow $c_{1}^{a}, c_{2}^{a}, c_{1}^{b}, c_{2}^{b}$ to share such mappings for all relevant edges $(D E)_{\rightarrow}$.

For edges $(F W)_{\rightarrow},(G W)_{\rightarrow}$ in Fig. $8(\mathrm{~b})$, we define $W(f, g)$ for $\{f, g\} \in$ $\mathfrak{X}_{\{F, G\}}=\{00,01,10,11\}$ in such a way that whenever $p_{1}^{*}(F, G) \neq p_{2}^{*}(F, G)$,

$$
\left(\sum_{f, g} p(W(f, g)) p_{1}^{*}(F=f, G=g)\right) \neq\left(\sum_{f, g} p(W(f, g)) p_{2}^{*}(F=f, G=g)\right)
$$

In other words, we define $p(W(f, g))$ in such a way that

$$
f_{(F W)_{\rightarrow,}(G W)_{\rightarrow}}\left(p^{*}(F, G)\right) \mapsto p^{*}(W):\left(\sum_{f, g} p(W(f, g)) p^{*}(F=f, G=g)\right)
$$

is a one to one linear map from distributions over $F, G$ to distributions over $W$. Such mappings clearly exist: it suffices to consider a 2 by 4 matrix with entries between 0 and 1, where all columns sum to 1 and are linearly independent. We allow $c_{1}^{b}, c_{2}^{b}$ to share such a mapping. As before, we interpret these 
mappings as independent imperative computer programs that are invariant under variable replacement, or as structural equations.

We now have to show that that the newly defined causal structures are in the SWM with respect to the appropriate subgraph of $\mathcal{G}$, agree on $p(\mathbf{V}(*))$, but disagree on the response to an appropriate edge intervention.

Call the set of all variables in our extended causal structures $\mathbf{V}$. We have to show that for all $\mathbf{v} \in \mathfrak{X}_{\mathbf{V}}$, the set $\left\{V\left(\mathbf{v}_{\mathrm{pa}_{\mathcal{G}}(V)}\right) \mid V \in \mathbf{V}\right\}$ is mutually independent. We have already shown mutual independence for potential outcomes derived from $A, B, C$ earlier. $V_{1}, \ldots V_{k}$ will be mutually independent of each other and any other variable by definition, as will $V\left(\mathbf{v}_{\mathrm{pa}_{\mathcal{G}}(V)}\right)$ for any $V \in \mathbf{V} \backslash\left\{A, B, C, V_{1}, \ldots V_{k}\right\}$, since all such potential outcomes are defined via mutually independent linear maps.

Fix $\mathbf{A} \subseteq \mathbf{V}, \mathbf{a} \in \mathfrak{X}_{\mathbf{A}}$, and a topological ordering $\prec$ on $\mathcal{G}$ such that $\{A, B, C\}$ occur before all other variables in $\mathbf{V}$ in the ordering. Then $p(\mathbf{V}(\mathbf{a})=$ v) is equal to

$$
\begin{aligned}
& p\left(\{\mathbf{V} \backslash\{A, B, C\}\}(\mathbf{a})=\mathbf{v}_{\mathbf{V} \backslash\{A, B, C\}} \mid\{A, B, C\}(\mathbf{a})=\mathbf{v}_{\{A, B, C\}}\right) . \\
& p\left(\{A, B, C\}(\mathbf{a})=\mathbf{v}_{\{A, B, C\}}\right) \\
& =\left(\prod_{V \in \mathbf{V} \backslash\{A, B, C\}} p\left(V(\mathbf{a})=\mathbf{v}_{V} \mid \operatorname{pre}_{\prec \mathcal{G}}(V)(\mathbf{a})=\mathbf{v}_{\text {pre }_{\prec_{\mathcal{G}}}(V)}\right)\right) . \\
& p\left(\{A, B, C\}(\mathbf{a})=\mathbf{v}_{\{A, B, C\}}\right) \\
& =\left(\prod_{V \in \mathbf{V} \backslash\{A, B, C\}} p\left(V(\mathbf{a})=\mathbf{v}_{V} \mid \operatorname{pa}_{\mathcal{G}}(V)(\mathbf{a})=\mathbf{v}_{\mathrm{pa}_{\mathcal{G}}(V)}\right)\right) . \\
& p\left(\{A, B, C\}(\mathbf{a})=\mathbf{v}_{\{A, B, C\}}\right) \\
& =\quad\left(\prod_{V \in \mathbf{V} \backslash\{A, B, C\}} p\left(V=\mathbf{v}_{V} \mid \mathbf{a}_{\mathrm{pa}_{\mathcal{G}}(V) \cap \mathbf{A}}, \mathbf{v}_{\mathrm{pa}_{\mathcal{G}}(V) \backslash \mathbf{A}}\right)\right) . \\
& p\left(\{A, B, C\}(\mathbf{a})=\mathbf{v}_{\{A, B, C\}}\right) \\
& =\left(\prod_{V \in \mathbf{V} \backslash\{A, B, C\}} p\left(V\left(\mathbf{a}_{\mathbf{p a}_{\mathcal{G}}(V) \cap \mathbf{A}}, \mathbf{v}_{\mathrm{pa}_{\mathcal{G}}(V) \backslash \mathbf{A}}\right)=\mathbf{v}_{V}\right)\right) \text {. } \\
& p\left(\{A, B, C\}(\mathbf{a})=\mathbf{v}_{\{A, B, C\}}\right)
\end{aligned}
$$

Here the first and second identities follow by the chain rule of probability, the other identities follow by the factorization and modularity properties of the SWMs, which hold because all structures in question are in the appropriate SWM for the graphs either in Fig. 8 (a) or (b).

Then, $c_{1}^{a}, c_{1}^{a}, c_{1}^{b}, c_{2}^{b}$ all agree on the term $p(\{A, B, C\}(\mathbf{a}))$, since this term 
is defined in the same way as in $c_{1}, c_{2}$ in lemma 0.8. All other terms are of the form $V\left(\mathbf{w}_{\mathrm{pa}_{\mathcal{G}}(V)}\right)$, for some $\mathbf{w} \in \mathfrak{X}_{\mathrm{pa}_{\mathcal{G}}(V)}$. But these structures all agree on how these terms are defined. Thus these structures all agree on $p(\mathbf{V}(\mathbf{a}))$.

Next, we show that $c_{1}^{a}$ and $c_{2}^{a}$ disagree on $p\left(\left\{Y_{1}, Y_{2}\right\}\left(\mathfrak{a}_{\left.(A B)_{\rightarrow},(A C)_{\rightarrow}\right)}\right)\right.$, and

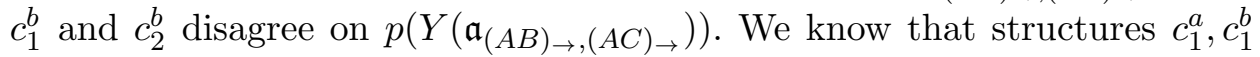

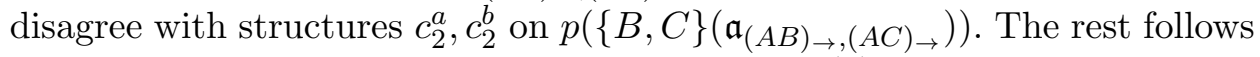
by induction on the paths $\alpha$ and $\beta$ for Fig. 8 (a), and on the paths $\alpha$, $\beta$ and $\gamma$ for Fig. 8 (b). The base case gives us two different distributions $p_{1}^{*}(b, c), p_{2}^{*}(b, c)$. For the inductive case, assume we have distinct $p_{1}^{*}(d, e)$ and $p_{2}^{*}(d, e)$, and either an edge $(E F) \rightarrow$ in Fig. 8 (a) or (b), or a pair of edges $(D W)_{\rightarrow},(E W)_{\rightarrow}$ in Fig. 8 (b). In either case, by definition, the one to one mapping for $F(e)$ or $W(d, e)$ ensures that

$$
\left(p_{1}^{\dagger}(d, f)=\sum_{e} p_{1}^{*}(d, e) p(F(e)=f)\right) \neq\left(p_{2}^{\dagger}(d, f)=\sum_{e} p_{2}^{*}(d, e) p(F(e)=f)\right)
$$

and, similarly,

$$
\left(p_{1}^{\dagger}(w)=\sum_{d, e} p_{1}^{*}(d, e) p(W(d, e)=w)\right) \neq\left(p_{2}^{\dagger}(w)=\sum_{d, e} p_{2}^{*}(d, e) p(W(d, e)=w)\right)
$$

This established that $p\left(\left\{Y_{1}, Y_{2}\right\}\left(\mathfrak{a}_{(A B)_{\rightarrow},(A C)_{\rightarrow}}\right)\right)$ is not identifiable in terms of $p(\mathbf{V}(*))$ under the SWM for Fig. $8\left(\right.$ a), and similarly for $p\left(Y\left(\mathfrak{a}_{(A B)_{\rightarrow},(A C)_{\rightarrow}}\right)\right)$ for Fig. 8 (a).

Since we assumed an edge intervention on a live set of edges for $\mathbf{Y}$, no edges in $\boldsymbol{\alpha} \backslash\left\{(A B)_{\rightarrow},(A C)_{\rightarrow}\right\}$ are in the graphs in Fig. 8 (a) or (b). This implies that under the SWM for the graph in (a), $p\left(\left\{Y_{1}, Y_{2}\right\}\left(\mathfrak{a}_{\boldsymbol{\alpha}}\right)\right)$ is not identifiable, and under the SWM for the graph in (b), $p\left(Y\left(\mathfrak{a}_{\boldsymbol{\alpha}}\right)\right)$ is not identifiable.

Recall that we originally needed to show $p\left(\mathbf{Y}\left(\mathfrak{a}_{\boldsymbol{\alpha}}\right)\right)$ was not identifiable from $\left\{p(\mathbf{V}(\mathbf{a})) \mid \mathbf{a} \in \mathfrak{X}_{\mathbf{A}}, \mathbf{A} \subseteq \mathbf{V}\right\}$ under the SWM for a DAG $\mathcal{G}$, of which the graphs in Fig. 8 (a) and (b) are edge subgraphs. But this follows by lemma 5.5.

Lemma 5.6. For a $D A G \mathcal{G}$ with vertices $\mathbf{V}$, and an edge intervention $\eta_{\mathfrak{a}_{\boldsymbol{\alpha}}}$ on an edge set $\boldsymbol{\alpha}$, we have, under the $M W M$ for $\mathcal{G}$,

$$
p\left(\mathbf{V}\left(\mathfrak{a}_{\boldsymbol{\alpha}}\right)=\mathbf{v}\right)=\prod_{V \in \mathbf{V}} p\left(V=\mathbf{v}_{V} \mid \mathbf{v}_{\mathrm{pa}_{\mathcal{G}}^{\bar{\alpha}}(V)}, \mathfrak{a}_{\left\{(W V)_{\rightarrow} \in \boldsymbol{\alpha}\right\}}\right)
$$


Proof. For any topological ordering $\prec$ of vertices in $\mathcal{G}, p\left(\mathbf{V}\left(\mathfrak{a}_{\boldsymbol{\alpha}}\right)=\mathbf{v}\right)=$

$$
\begin{aligned}
& =\prod_{V \in \mathbf{V}} p\left(V\left(\mathfrak{a}_{\boldsymbol{\alpha}}\right)=\mathbf{v}_{V} \mid \operatorname{pre}_{\prec \mathcal{G}}(V)\left(\mathfrak{a}_{\boldsymbol{\alpha}}\right)=\mathbf{v}_{\mathrm{pre}_{\prec \mathcal{G}}(V)}\right) \\
& =\prod_{V \in \mathbf{V}} p\left(V\left(\mathbf{v}_{\mathrm{pa}_{\mathcal{G}}^{\bar{\alpha}}(V)}, \mathfrak{a}_{\left\{(W V)_{\rightarrow} \in \boldsymbol{\alpha}\right\}}\right)=\mathbf{v}_{V} \mid\right. \\
& \left.\quad\left\{W\left(\mathbf{v}_{\mathrm{pa}_{\mathcal{G}}^{\bar{\alpha}}(W)}, \mathfrak{a}_{\left\{(Z W)_{\rightarrow} \in \boldsymbol{\alpha}\right\}}\right)=\mathbf{v}_{W} \mid W \in \operatorname{pre}_{\prec \mathcal{G}}(V)\right\}\right) \\
& =\prod_{V \in \mathbf{V}} p\left(V\left(\mathbf{v}_{\operatorname{pa}_{\mathcal{G}}^{\bar{\alpha}}(V)}, \mathfrak{a}_{\left\{(W V)_{\rightarrow} \in \boldsymbol{\alpha}\right\}}\right)=\mathbf{v}_{V}\right) \\
& =\prod_{V \in \mathbf{V}} p\left(V=\mathbf{v}_{V} \mid \mathbf{v}_{\mathrm{pa}_{\mathcal{G}}^{\bar{\alpha}}(V)}, \mathfrak{a}_{\left\{(W V)_{\rightarrow} \in \boldsymbol{\alpha}\right\}}\right)
\end{aligned}
$$

The first identity follows by the chain rule of probability, the second by definition of (3), the third by the independence assumption of the MWM for $\mathcal{G}$, and the last by properties of the SWM described in [3], which also hold in the MWM since it is a submodel of the SWM.

Lemma 5.7. Given a $D A G \mathcal{G}$ with vertices $\mathbf{V}$, and a path intervention $\pi_{\mathfrak{a}_{\boldsymbol{\alpha}}}$ edge consistent for $\mathbf{Y} \subseteq \mathbf{V}, p\left(\mathbf{Y}\left(\mathfrak{a}_{\boldsymbol{\alpha}}\right)\right)=p\left(\mathbf{Y}\left(\mathfrak{a}_{\boldsymbol{\alpha}_{1}}\right)\right)$.

Proof. Fix $\beta \in \operatorname{rel}_{\mathcal{G}}(\mathbf{Y} \mid \boldsymbol{\alpha})$ such that $\beta$ has a prefix $\alpha \in \boldsymbol{\alpha}$. Since $\alpha$ is the only subpath in $\alpha$ in $\beta$, either the edge prefix $\alpha_{1}$ of $\alpha$ is the only element of $\boldsymbol{\alpha}_{1}$ in $\beta$, or there is another edge in $\boldsymbol{\alpha}_{1}$ in $\beta$. The latter implies $\pi$ is edge inconsistent, which is a contradiction. The former implies $\beta \in \operatorname{rel}_{\mathcal{G}}\left(\mathbf{Y} \mid \boldsymbol{\alpha}_{1}\right)$ with an edge prefix in $\boldsymbol{\alpha}_{1}$.

Fix $\beta \in \operatorname{rel}_{\mathcal{G}}(\mathbf{Y} \mid \boldsymbol{\alpha})$ such that $\beta$ contains no element in $\boldsymbol{\alpha}$, but has a parent-less source. Assume $\beta$ contains an element $\alpha_{1} \in \boldsymbol{\alpha}_{1}$. By definition of $\boldsymbol{\alpha}_{1}$, this implies $\alpha_{1}$ is an edge prefix of a path in $\operatorname{rel}_{\mathcal{G}}(\mathbf{Y} \mid \boldsymbol{\alpha})$ with a prefix in $\boldsymbol{\alpha}$. But $\alpha_{1}$ is also an element of $\beta \in \operatorname{rel}_{\mathcal{G}}(\mathbf{Y} \mid \boldsymbol{\alpha})$ which does not contain a prefix subpath in $\boldsymbol{\alpha}$. This contradicts our assumption that $\pi$ is edge consistent for $\mathbf{Y}$. Thus $\beta \in \operatorname{rel}_{\mathcal{G}}\left(\mathbf{Y} \mid \boldsymbol{\alpha}_{1}\right)$, has a parent-less source, and contains no elements of $\boldsymbol{\alpha}_{1}$.

Fix $\beta \in \operatorname{rel}_{\mathcal{G}}\left(\mathbf{Y} \mid \boldsymbol{\alpha}_{1}\right)$ such that $\beta$ has an edge prefix $\alpha_{1} \in \boldsymbol{\alpha}_{1}$. This implies $\beta$ does not contain any elements in $\boldsymbol{\alpha}_{1}$ other than $\alpha_{1}$. Furthermore, by definition of $\boldsymbol{\alpha}_{1}, \alpha_{1}$ is an edge prefix of some $\alpha \in \boldsymbol{\alpha}$. This implies that if $\beta$ contains elements of $\boldsymbol{\alpha}$ at all, there can only be one, and it must be a prefix subpath of $\beta$. This would imply $\beta \in \operatorname{rel}_{\mathcal{G}}(\mathbf{Y} \mid \boldsymbol{\alpha})$. If $\beta$ contains no elements in $\boldsymbol{\alpha}$, then $\beta$ is a suffix of a path $\gamma \in \operatorname{rel}_{\mathcal{G}}(\mathbf{Y} \mid \boldsymbol{\alpha})$ which contains $\alpha_{1}$, and $\alpha_{1}$ is not an edge prefix of a prefix subpath of $\gamma$ that lies in $\boldsymbol{\alpha}$. But this contradicts our assumption that $\pi$ is edge consistent for $\mathbf{Y}$. 
Fix $\beta \in \operatorname{rel}_{\mathcal{G}}\left(\mathbf{Y} \mid \boldsymbol{\alpha}_{1}\right)$ such that $\beta$ contains no elements of $\boldsymbol{\alpha}_{1}$, and has a parent-less source. This implies $\beta$ contains no elements of $\alpha$. Thus $\beta \in$ $\operatorname{rel}_{\mathcal{G}}(\mathbf{Y} \mid \boldsymbol{\alpha})$, has a parent-less source, and contains no elements of $\boldsymbol{\alpha}$.

We have thus established a bijective correspondence between paths in $\operatorname{rel}_{\mathcal{G}}(\mathbf{Y} \mid \boldsymbol{\alpha})$ and $\operatorname{rel}_{\mathcal{G}}\left(\mathbf{Y} \mid \boldsymbol{\alpha}_{1}\right)$ that either contain no elements of $\boldsymbol{\alpha}\left(\boldsymbol{\alpha}_{1}\right)$ and have parent-less sources, or contain a prefix in $\boldsymbol{\alpha}\left(\boldsymbol{\alpha}_{1}\right)$. These are precisely the paths that correspond to paths from leaf to root in $\mathcal{F}(\mathbf{Y}, \boldsymbol{\alpha}, \mathcal{G})$ and $\mathcal{F}\left(\mathbf{Y}, \boldsymbol{\alpha}_{1}, \mathcal{G}\right)$. That the assignments to sources of these paths correspond in both $\pi_{\mathfrak{a}_{\alpha}}$ and $\eta_{\mathfrak{a}_{\alpha_{1}}}$ follows by definition of $\eta$.

Lemma 5.8. The distributions $p\left(B(a), B\left(a^{\prime}\right)\right)$ and $p(B(a), B)$ are not identifiable from $p(A, B)$ under the $M W M$ for the DAG in Fig. 3 (a).

Proof. A proof of this appears in [1]. We reproduce the proof here to better connect with the language of causal structures used in this manuscript, and to facilitate the extension of the causal structures used in the proof for the purposes of other proofs later.

Consider the following two sets of random variables $\mathbf{V}_{1}$ and $\mathbf{V}_{2}$ associated with vertices $\mathbf{V}=\left\{A, B, U_{B}\right\}$ as follows:

$$
\begin{array}{llll} 
& A & U_{B} & B \\
\mathbf{V}_{1} & \operatorname{unif}(\{0,1\}) & \operatorname{unif}(\{0,1,2\}) & \mathbb{I}\left(U_{B} \neq 0\right) \oplus A \\
\mathbf{V}_{2} & \operatorname{unif}(\{0,1\}) & \operatorname{unif}(\{0,1,2\}) & \mathbb{I}\left(U_{B}=0 \vee\left(U_{B}=1 \wedge A=0\right)\right)
\end{array}
$$

where $\mathbb{I}$ (condition) is the indicator function which evaluates to 1 if the condition holds, and to 0 otherwise.

As before, we define two causal structures $c_{1}\left(\{A, B\}, \mathcal{G}_{1}\right), c_{2}\left(\{A, B\}, \mathcal{G}_{1}\right)$, where $\mathcal{G}_{1}$ is the graph in Fig. 3 (a), by interpreting these definitions as structural equations where variable replacement is possible, and considering joint distributions over the resulting potential outcomes induced by $p\left(U_{B}\right)$. In other words, we have $A=A(a)=A(b)=\operatorname{unif}(\{0,1\})$ for all $a, b \in \mathfrak{X}_{\{A, B\}}$ in both $c_{1}$ and $c_{2}$, while $B(a)=B(a, b)=\operatorname{Binom}(1,2 / 3)$ and $B\left(a^{\prime}\right)=$ $B\left(a^{\prime}, b\right)=\operatorname{binom}(1,1 / 3)$ for all $b \in \mathfrak{X}_{B}$ in both $c_{1}$ and $c_{2}$.

Since $U_{B}$ and $A$ were assumed to be independent, we have $A \Perp B(a)$ and $A \Perp B\left(a^{\prime}\right)$ in both structures. Thus, both $c_{1}\left(\{A, B\}, \mathcal{G}_{1}\right)$ and $c_{2}\left(\{A, B\}, \mathcal{G}_{1}\right)$ are in the SWM for $\mathcal{G}_{1}$, and moreover agree on $\left\{p\left(\{A, B\}(\mathbf{w}) \mid \mathbf{w} \in \mathfrak{X}_{\mathbf{W}}, \mathbf{W} \subseteq\right.\right.$ $\{A, B\}\}$.

Our definition of $c_{1}$ and $c_{2}$ now induce the following table (assuming $\left.a=1, a^{\prime}=0\right)$ : 


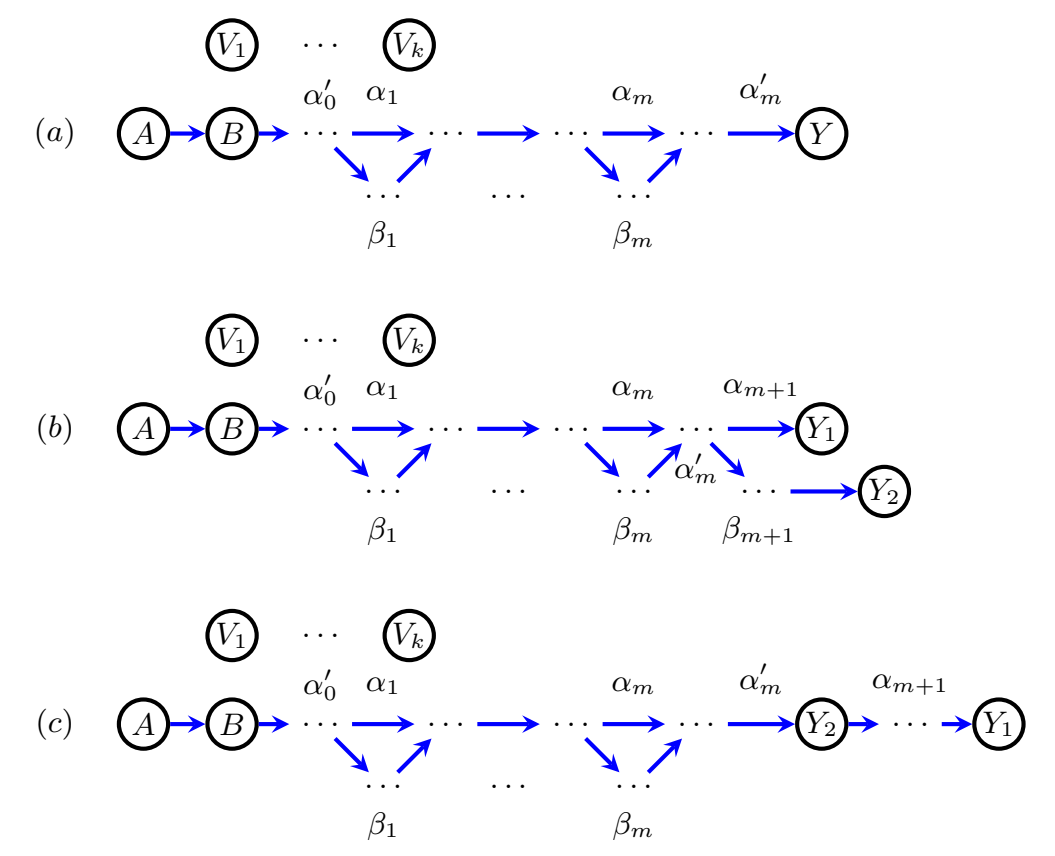

Fig 9. A schematic representation of graphs used in the proof of theorem 5.2.

\begin{tabular}{c|ccc|ccc}
$U_{B}$ & $B(a)_{c_{1}}$ & $B\left(a^{\prime}\right)_{c_{1}}$ & $B_{c_{1}}$ & $B(a)_{c_{2}}$ & $B\left(a^{\prime}\right)_{c_{2}}$ & $B_{c_{2}}$ \\
\hline 0 & 1 & 0 & $\operatorname{unif}(\{0,1\})$ & 1 & 1 & 1 \\
1 & 0 & 1 & $\operatorname{unif}(\{0,1\})$ & 0 & 1 & $\operatorname{unif}(\{0,1\})$ \\
2 & 0 & 1 & $\operatorname{unif}(\{0,1\})$ & 0 & 0 & 0
\end{tabular}

where each row has probability $1 / 3$ of occurring. Our conclusion directly follows.

Theorem 5.2. Consider a DAGG with vertices $\mathbf{V}$, and a proper set of paths $\boldsymbol{\alpha}$ live for $\mathbf{Y}$. Then $p\left(\mathbf{Y}\left(\mathfrak{a}_{\boldsymbol{\alpha}}\right)\right)$ is identifiable from $p(\mathbf{V})$ under the $M W M$ for $\mathcal{G}$ if and only if $\pi_{\mathfrak{a}_{\boldsymbol{\alpha}}}$ is edge consistent. Moreover, if $p\left(\mathbf{Y}\left(\mathfrak{a}_{\boldsymbol{\alpha}}\right)\right)$ is identifiable, it is equal to the appropriate marginal of the edge g-formula for $p\left(\mathbf{Y}\left(\mathfrak{a}_{\boldsymbol{\alpha}_{1}}\right)\right)$, the response to the induced edge intervention.

Proof. If $\pi_{\mathfrak{a}_{\alpha}}$ is edge consistent, then identification follows by corollary 5.2 .

If $\pi_{\mathfrak{a}_{\boldsymbol{\alpha}}}$ is edge inconsistent, either $\boldsymbol{\alpha}$ is consistent for $\mathbf{Y}$ but there exist two distinct directed paths $\alpha, \beta \in \operatorname{rel}_{\mathcal{G}}(\mathbf{Y} \mid \boldsymbol{\alpha})$ with a shared edge prefix $(A B)_{\rightarrow}$, and path prefixes $\alpha^{*}, \beta^{*} \in \boldsymbol{\alpha}$ assigned different values of $A$ by $\pi$, 
or $\boldsymbol{\alpha}$ is not consistent for $\mathbf{Y}$. That is, $(A B)_{\rightarrow}$ is an edge prefix of $\alpha^{*} \in \boldsymbol{\alpha}$ with a corresponding path $\alpha \in \operatorname{rel}_{\mathcal{G}}(\mathbf{Y} \mid \boldsymbol{\alpha})$, and there exists another path $\beta \in \operatorname{rel}_{\mathcal{G}}(\mathbf{Y} \mid \boldsymbol{\alpha})$ that contains $(A B) \rightarrow$ but either $\beta$ does not contain any elements of $\boldsymbol{\alpha}$ or $(A B)_{\rightarrow}$ is not an edge prefix of $\beta$.

In the former case, we will include all of both $\alpha$ and $\beta$. In the latter case, we will include all of $\alpha$ and a suffix subpath of $\beta$ that starts with $(A B)_{\rightarrow}$. We will consider three cases, shown schematically in Fig. 9 (a), (b) and (c). In the first case $\operatorname{si}_{\mathcal{G}}(\{\alpha, \beta\})=\{Y\}$, in the second and third cases $\operatorname{si}_{\mathcal{G}}(\{\alpha, \beta\})=\left\{Y_{1}, Y_{2}\right\}$. In the second case the path ending in $Y_{1}$ is $\alpha$, and the path ending in $Y_{2}$ is $\beta$. In the third case, the same is true, but (without loss of generality) $Y_{2}$ is an ancestor of $Y_{1}$ along a path segment of $\alpha$.

We subdivide $\alpha$ and $\beta$ into segments as follows. $\beta$ is subdivided into $\beta_{1}, \ldots \beta_{m}$, such that the source and sink of each $\beta_{i},(i=1, \ldots m)$ are in $\alpha$, but no intermediate node is in $\alpha$. Furthermore, in case (b), we also consider $\beta_{m+1}$ with the source the last element in common between $\alpha$ and $\beta$, and the sink $Y_{2}$. In cases (a) and (c), $m>0$, while it is possible in case (b) that $m=0$.

$\alpha$ is subdivided into $(A B)_{\rightarrow}, \alpha_{0}^{\prime}, \alpha_{1}, \alpha_{1}^{\prime}, \ldots \alpha_{m}, \alpha_{m}^{\prime}, \alpha_{m+1}$, where $\alpha_{0}^{\prime}$ is a segment with source $C$ to the first vertex at which the paths $\alpha$ and $\beta$ deviate, $\alpha_{i}$ is the segment with the same source and sink as $\beta_{i}, \alpha_{i}^{\prime}$ has the source equal to the sink of $\beta_{i}$, and the sink equal to the source of $\beta_{i+1}$, and $\alpha_{m+1}$ is the segment in cases (b) and (c) with $Y_{1}$ as a sink, and the last node in common between $\beta$ and $\alpha$ as a source. This subdivision is also shown in Fig. 9 .

By assumption, all graphs in Fig. 9 are edge subgraphs of the DAG $\mathcal{G}$. In particular, $\left\{V_{1}, \ldots V_{k}\right\}$ is the set of vertices in $\mathbf{V}$ that do not appear in either $\alpha$ or $\beta$. We are going to extend the pair of causal structures $c_{1}, c_{2}$ which appear in the proof of lemma 5.8 to pairs of causal structures $c_{1}^{a}, c_{2}^{a}$, $c_{1}^{b}, c_{2}^{b}$ and $c_{1}^{c}, c_{2}^{c}$ associated with an MWM for graphs in Fig. 9 (a), (b), and (c), respectively. We will use binary state space models - the result easily generalizes to arbitrary state spaces.

We will use the same causal structure extension we used in the proof of theorem 5.1. Specifically, for every node $E$ in the graphs in Fig. 9 with a single parent $D$, we define $E(d)$ (for all $d \in \mathfrak{X}_{D}$ ), as the same one to one linear mapping in $c_{1}^{*}$ and $c_{2}^{*}$. For every $E$ in the graphs in Fig. 9 with two parents $C, D$, we define $E(c, d)$ (for all $\{c, d\} \in \mathfrak{X}_{\{C, D\}}$ ), as the same one to one linear mapping in $c_{1}^{*}$ and $c_{2}^{*}$.

In case 1 , we have to show $p\left(Y\left(\mathfrak{a}_{\{\alpha, \beta\}}\right)\right)\left(\right.$ or $\left.p\left(Y\left(\mathfrak{a}_{\{\alpha\}}\right)\right)\right)$ is not identifiable, and in cases 2 and 3 , we have to show $p\left(\left\{Y_{1}, Y_{2}\right\}\left(\mathfrak{a}_{\{\alpha, \beta\}}\right)\right)\left(\right.$ or $\left.p\left(\left\{Y_{1}, Y_{2}\right\}\left(\mathfrak{a}_{\{\alpha\}}\right)\right)\right)$ is not identifiable. 
The proof proceeds by induction on the paths $\alpha$ and $\beta$, in a way similar to the proof of theorem 5.1. Our base case is the two causal structures $c_{1}^{*}$ and $c_{2}^{*}$ disagree on $p\left(B(a), B\left(a^{\prime}\right)\right)$ or $p(B(a), B)$. We have a number of inductive cases to consider. We list them all for completeness, but the proof in each case follows by the fact that the local maps relevant for each case are one to one linear maps.

A single inductive step has the following cases, where we start with a distribution over intermediate responses on which $c_{1}^{*}$ and $c_{2}^{*}$ disagree, and a set of edges and associated one to one linear mapping which translates the disagreement into the next step. We illustrate the cases with a table. Note that while there are many cases, the induction takes the same form in all of them. We define $\alpha_{W}$ to be a segment of $\alpha$ with the same source as $\alpha$, but with sink at $W$. Below, $i$ ranges from 1 to $m$, and $j$ from 0 to $m$.

$$
\begin{array}{ccc}
\text { starting response } & \text { edge set } & \text { ending response } \\
p\left(C\left(\mathfrak{a}_{\alpha_{C}}\right), C\left(\mathfrak{a}_{\beta_{C}}\right)\right) & (C D)_{\rightarrow} \in \alpha_{j}^{\prime} & p\left(D\left(\mathfrak{a}_{\alpha_{D}}\right), D\left(\mathfrak{a}_{\beta_{D}}\right)\right) \\
p\left(C\left(\mathfrak{a}_{\alpha_{C}}\right), C\left(\mathfrak{a}_{\beta_{C}}\right)\right) & (C D)_{\rightarrow} \in \alpha_{i},(C E)_{\rightarrow} \in \beta_{i} & p\left(D\left(\mathfrak{a}_{\alpha_{D}}\right), E\left(\mathfrak{a}_{\beta_{E}}\right)\right) \\
p\left(D\left(\mathfrak{a}_{\alpha_{D}}\right), E\left(\mathfrak{a}_{\beta_{E}}\right)\right) & (D F)_{\rightarrow} \in \alpha_{j+1} & p\left(F\left(\mathfrak{a}_{\alpha_{F}}\right), E\left(\mathfrak{a}_{\beta_{E}}\right)\right) \\
p\left(D\left(\mathfrak{a}_{\alpha_{D}}\right), E\left(\mathfrak{a}_{\beta_{E}}\right)\right) & (E F)_{\rightarrow} \in \beta_{i} & p\left(D\left(\mathfrak{a}_{\alpha_{D}}\right), F\left(\mathfrak{a}_{\beta_{F}}\right)\right) \\
p\left(D\left(\mathfrak{a}_{\alpha_{D}}\right), E\left(\mathfrak{a}_{\beta_{E}}\right)\right) & (D W)_{\rightarrow} \in \alpha_{i},(E W)_{\rightarrow} \in \beta_{i} & p\left(W\left(\mathfrak{a}_{\alpha_{W}}\right), W\left(\mathfrak{a}_{\beta_{W}}\right)\right) \\
p\left(D\left(\mathfrak{a}_{\alpha_{D}}\right), E\left(\mathfrak{a}_{\beta_{E}}\right)\right) & (D W)_{\rightarrow} \in \alpha_{m},(E W)_{\rightarrow} \in \beta_{m}, & p\left(W\left(\mathfrak{a}_{\left\{\alpha_{W}, \beta_{W}\right\}}\right)\right) \\
p\left(C\left(\mathfrak{a}_{\left\{\alpha_{C}, \beta_{C}\right\}}\right)\right. & (C D)_{\rightarrow} \in(C \ldots Y)_{\rightarrow} & p\left(D\left(\mathfrak{a}_{\left\{\alpha_{D}, \beta_{D}\right\}}\right)\right) \\
p\left(Y_{2}\left(\mathfrak{a}_{\beta}\right), C\left(\mathfrak{a}_{\alpha_{C}}\right)\right) & (C D)_{\rightarrow} \in\left(Y_{2} \ldots Y_{1}\right)_{\rightarrow} & p\left(Y_{2}\left(\mathfrak{a}_{\beta}\right), D\left(\mathfrak{a}_{\alpha_{D}}\right)\right)
\end{array}
$$

Here the edge set in the 6th case refers to case (a) in Fig. 9.

This establishes that the causal structures $c_{1}^{a}, c_{1}^{a}$ disagree on the relevant response to our path intervention in all cases under the MWM associated with graphs in Fig. 9.

Recall that we originally needed to show $p\left(\mathbf{Y}\left(\mathfrak{a}_{\boldsymbol{\alpha}}\right)\right)$ was not identifiable from $\left\{p(\mathbf{V}(\mathbf{a})) \mid \mathbf{a} \in \mathfrak{X}_{\mathbf{A}}, \mathbf{A} \subseteq \mathbf{V}\right\}$ under the MWM for a DAG $\mathcal{G}$, of which the graphs in Fig. 9 (a), (b), (c) are edge subgraphs. But this follows by lemma 5.5

\section{Proofs for Section 7.}

Lemma 7.1. If $\left(\forall \mathbf{S}_{1}, \mathbf{S}_{2} \in \mathcal{D}\left(\mathcal{G}_{\mathbf{V} \backslash \mathbf{A}}\right)\right)\left(\mathbf{D}_{\mathbf{S}_{1}} \cap \mathbf{D}_{\mathbf{S}_{2}} \neq \emptyset\right) \Rightarrow\left(\mathbf{S}_{1}=\mathbf{S}_{2}\right)$, then the sets $\left\{\mathbf{D}_{\mathbf{S}} \mid \mathbf{S} \in \mathcal{D}\left(\mathcal{G}_{\mathbf{V} \backslash \mathbf{A}}\right)\right\}$ partition $\mathbf{V} \backslash \mathbf{A}_{f}$.

Proof. If $V \in \mathbf{V} \backslash \mathbf{A}$, then since $\mathcal{D}\left(\mathcal{G}_{\mathbf{V} \backslash \mathbf{A}}\right)$ partitions $\mathbf{V} \backslash \mathbf{A}$, there exists $\mathbf{S} \in \mathcal{D}\left(\mathcal{G}_{\mathbf{V} \backslash \mathbf{A}}\right)$ such that $V \in \mathbf{S}$. Thus $V \in \mathbf{D}_{\mathbf{S}}$. If $V \in \mathbf{A} \backslash \mathbf{A}_{f}, V \in$ $\bigcup_{\mathbf{S} \in \mathcal{D}\left(\mathcal{G}_{\mathbf{V} \backslash \mathbf{A}}\right)} \mathbf{D}_{\mathbf{S}}$. If $V \in \mathbf{D}_{\mathbf{S}_{1}} \cap \mathbf{D}_{\mathbf{S}_{2}}$, for some $\mathbf{S}_{1}, \mathbf{S}_{2} \in \mathcal{D}\left(\mathcal{G}_{\mathbf{V} \backslash \mathbf{A}}\right)$, then by assumption, $\mathbf{S}_{1}=\mathbf{S}_{2}$. This gives our result. 
Lemma 7.2. Fix $\mathbf{A}, \mathbf{Y}, \mathcal{G}$ such that

$1\left(\forall \mathbf{S} \in \mathcal{D}\left(\mathcal{G}_{\mathbf{V} \backslash \mathbf{A}}\right)\right),\left(\operatorname{pa}_{\mathcal{G}}(\mathbf{S}) \backslash \mathbf{S}\right) \cap \mathbf{D}_{\mathbf{S}}=\emptyset$, and

2 $\left(\forall \mathbf{S}_{1}, \mathbf{S}_{2} \in \mathcal{D}\left(\mathcal{G}_{\mathbf{V} \backslash \mathbf{A}}\right)\right),\left(\mathbf{D}_{\mathbf{S}_{1}} \cap \mathbf{D}_{\mathbf{S}_{2}} \neq \emptyset\right) \Rightarrow\left(\mathbf{S}_{1}=\mathbf{S}_{2}\right)$.

Then $p(\mathbf{Y}(\mathbf{a})=\mathbf{y})$ is identified by a g-functional

$$
\sum_{\left.\mathbf{v} \backslash \mathbf{V} \backslash \mathbf{Y} \cup \mathbf{A}_{f}\right)} \prod_{V \in \mathbf{V} \backslash \mathbf{A}_{f}} p\left((\mathbf{y} \cup \mathbf{v})_{V} \mid \mathbf{a}_{\operatorname{pre}_{\prec_{\mathcal{G}}}(V) \cap\left(\mathbf{A} \backslash \mathbf{D}_{V}\right)},(\mathbf{y} \cup \mathbf{v})_{\operatorname{pre}_{\prec_{\mathcal{G}}}(V) \backslash\left(\mathbf{A} \backslash \mathbf{D}_{V}\right)}\right) .
$$

Proof. We have that $p(\mathbf{Y}(\mathbf{a}))$ is equal to

$$
\begin{aligned}
& \sum_{\mathbf{V} \backslash(\mathbf{Y} \cup \mathbf{A})} \prod_{\mathbf{S} \in \mathcal{D}\left(\mathcal{G}_{\mathbf{V} \backslash \mathbf{A}}\right)} p\left(\mathbf{S}\left(\mathbf{a}_{\mathbf{A} \cap\left(\mathrm{pa}_{\mathcal{G}}(\mathbf{S}) \backslash \mathbf{S}\right)},\left\{\mathrm{pa}_{\mathcal{G}}(\mathbf{S}) \backslash(\mathbf{S} \cup \mathbf{A})\right\}\right)\right)
\end{aligned}
$$

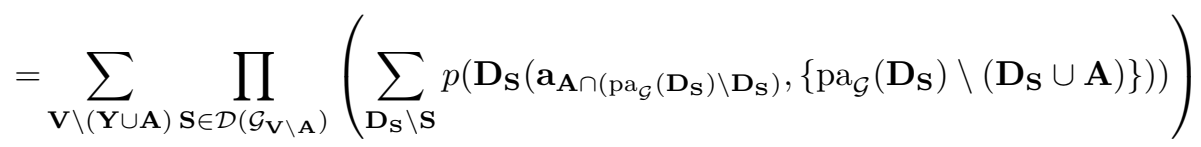

$$
\begin{aligned}
& =\sum_{\mathbf{V} \backslash(\mathbf{Y} \cup \mathbf{A})} \prod_{\mathbf{S} \in \mathcal{D}\left(\mathcal{G}_{\mathbf{V} \backslash \mathbf{A}}\right)}\left(\sum_{\mathbf{D}_{\mathbf{S}} \backslash \mathbf{S}} \prod_{V \in \mathbf{D}_{\mathbf{S}}} p\left(V \mid \mathbf{a}_{\operatorname{pre}_{\prec_{\mathcal{G}}}(V) \cap\left(\mathbf{A} \backslash \mathbf{D}_{\mathbf{S}}\right)}, \operatorname{pre}_{\prec_{\mathcal{G}}}(V) \backslash\left(\mathbf{A} \backslash \mathbf{D}_{\mathbf{S}}\right)\right)\right) \\
& =\sum_{\mathbf{V} \backslash\left(\mathbf{Y} \cup \mathbf{A}_{f}\right)} \prod_{V \in \mathbf{V} \backslash \mathbf{A}_{f}} p\left(V \mid \mathbf{a}_{\text {pre }_{\prec \mathcal{G}}(V) \cap\left(\mathbf{A} \backslash \mathbf{D}_{V}\right)}, \operatorname{pre}_{\prec \mathcal{G}}(V) \backslash\left(\mathbf{A} \backslash \mathbf{D}_{V}\right)\right)
\end{aligned}
$$

The first equality follows by standard results on district factorization in hidden variable causal models [4].

The second equality follows by assumption 2 , standard results on district factorization of hidden variable causal models (with the slight technical modification where variables are allowed to be both fixed and random, see [3] for details).

The third equality follows by assumption 1, standard results on causal effect identification, and the global Markov property of hidden variable DAGs.

The fourth equality follows by assumption 2. In particular, assumption 2 implies that $\left\{\mathbf{D}_{\mathbf{S}} \mid \mathbf{S} \in \mathcal{D}\left(\mathcal{G}_{\mathbf{V} \backslash \mathbf{A}}\right)\right\}$ partition $\mathbf{V} \backslash \mathbf{A}_{f}$ by Lemma 7.1. This implies we can bring the inner sums out, which gives our conclusion.

Lemma 7.3. For an $A D M G \mathcal{G}$ with vertex set $\mathbf{V}$, fix disjoint $\mathbf{Y}, \mathbf{A} \subseteq \mathbf{V}$ that satisfy the preconditions of lemma 7.2. Then there exists a DAG $\mathcal{G}^{\dagger}$ with vertex set $\mathbf{V}$, and an edge intervention $\eta_{\mathfrak{a}_{\boldsymbol{\alpha}}}$ on a set of edges $\boldsymbol{\alpha}$ in $\mathcal{G}^{\dagger}$ such that $p\left(\mathbf{Y}\left(\mathfrak{a}_{\boldsymbol{\alpha}}\right)\right)$ is identified under the $M W M$ for $\mathcal{G}^{\dagger}$ via a margin of the functional in (5) that is equal to the identifying g-functional for $p(\mathbf{Y}(\mathbf{a}))$ in terms of $p(\mathbf{V})$ in $\mathcal{G}$. 
Proof. Let $\mathcal{G}^{\dagger}$ be a DAG obtained from $\mathcal{G}$ by replacing all bidirected arrows by directed arrows consistent with $\prec$. Define $\boldsymbol{\alpha}$ to be all directed edges outgoing from $\mathbf{A}$ in $\mathcal{G}$, and let $\eta_{\mathfrak{a}_{\alpha}}$ assign any element $(A *) \rightarrow$ in $\boldsymbol{\alpha}$ to the value $\mathbf{a}_{A}$. Let $\mathbf{A}_{f}=\mathbf{A} \backslash\left(\bigcup_{V \in \mathbf{V} \backslash \mathbf{A}} \operatorname{dis}_{\mathcal{G}}(V)\right)$.

By lemma 7.2 and our assumptions, (2) is the identifying functional for $p(\mathbf{Y}(\mathbf{a}))$ in $\mathcal{G}$. We then get the following derivation

$$
\begin{aligned}
& \sum_{\mathbf{v}_{\mathbf{V} \backslash\left(\mathbf{Y} \cup \mathbf{A}_{f}\right)}} \prod_{V \in \mathbf{V} \backslash \mathbf{A}_{f}} p\left((\mathbf{y} \cup \mathbf{v})_{V} \mid(\mathbf{y} \cup \mathbf{v})_{\operatorname{pre}_{\prec_{\mathcal{G}}}(V) \backslash\left(\mathbf{A} \backslash \mathbf{D}_{V}\right)}, \mathbf{a}_{\mathrm{pre}_{\prec_{\mathcal{G}}}(V) \cap\left(\mathbf{A} \backslash \mathbf{D}_{V}\right)}\right) \\
= & \sum_{\mathbf{v}_{\mathbf{V} \backslash\left(\mathbf{Y} \cup \mathbf{A}_{f}\right)}} \prod_{V \in \mathbf{V} \backslash \mathbf{A}_{f}} p\left((\mathbf{y} \cup \mathbf{v})_{V} \mid(\mathbf{y} \cup \mathbf{v})_{\mathrm{pa}_{\mathcal{G}^{\dagger}}(V) \backslash\left(\mathbf{A} \backslash \mathbf{D}_{V}\right)}, \mathbf{a}_{\mathrm{pa}_{\mathcal{G}^{\dagger}}(V) \cap\left(\mathbf{A} \backslash \mathbf{D}_{V}\right)}\right) \\
= & \sum_{\mathbf{v}_{\mathbf{V} \backslash\left(\mathbf{Y} \cup \mathbf{A}_{f}\right)}} \prod_{V \in \mathbf{V} \backslash \mathbf{A}_{f}} p\left((\mathbf{y} \cup \mathbf{v})_{V} \mid(\mathbf{y} \cup \mathbf{v})_{\mathbf{p a}_{\mathcal{G}^{\dagger}}^{\bar{\alpha}}(V)}, \mathfrak{a}_{\left\{(W V)_{\rightarrow} \in \boldsymbol{\alpha}\right\}}\right) \\
= & \sum_{\mathbf{v}_{\mathbf{V} \backslash \mathbf{Y}}} \prod_{V \in \mathbf{V}} p\left((\mathbf{y} \cup \mathbf{v})_{V} \mid(\mathbf{y} \cup \mathbf{v})_{\operatorname{pa}_{\mathcal{G}^{\dagger}}^{\bar{\alpha}}(V)}, \mathfrak{a}_{\left\{(W V)_{\rightarrow} \in \boldsymbol{\alpha}\right\}}\right)
\end{aligned}
$$

which is the identifying functional for $p\left(\mathbf{Y}\left(\mathfrak{a}_{\boldsymbol{\alpha}}\right)=\mathbf{y}\right)$ in $\mathcal{G}^{*}$ as a direct consequence of (5).

To see that the first equality follows, note that $\operatorname{pa}_{\mathcal{G}^{\dagger}}(V) \subseteq \operatorname{pre}_{\prec_{\mathcal{G}^{\dagger}}}(V)=$ $\operatorname{pre}_{\prec_{\mathcal{G}}}(V)$ for any $V$ in $\mathcal{G}^{\dagger}$, and $p\left(V \mid \operatorname{pa}_{\mathcal{G}^{\dagger}}(V)\right)=p\left(V \mid \operatorname{pre}_{\prec_{\mathcal{G}^{\dagger}}}(V)\right)$ by the ordered local Markov property for $\mathcal{G}^{\dagger}$.

Any element in $\operatorname{pa}_{\mathcal{G}^{\dagger}}(V) \cap\left(\mathbf{A} \backslash \mathbf{D}_{V}\right)$ must be a parent of $V$ along an edge $\alpha$ in $\boldsymbol{\alpha}$. Consider $W \in \mathrm{pa}_{\mathcal{G}^{\dagger}}(V) \backslash\left(\mathbf{A} \backslash \mathbf{D}_{V}\right)$, which is a parent of $V$ along an edge $\beta$. If $W \notin \mathbf{A}, \beta \notin \boldsymbol{\alpha}$. If $W \in \mathbf{A}, W \in \mathbf{D}_{V}$. Then either $\beta$ is not in $\mathcal{G}$, which implies $\beta \notin \boldsymbol{\alpha}$, or our assumptions on input are violated. This implies the second equality.

To see that the third equality follows, note that the definition of $\eta$ and $\mathbf{A}_{f}$ is such that for any $A \in \mathbf{A}_{f}$, the term corresponding to any child of $A$ in the first equation involves the constant $\mathbf{a}_{A}$. Thus, all terms corresponding to elements in $\mathbf{A}_{f}$, and the corresponding marginalization of those terms can safely be added to the expression.

\section{References.}

[1] Chen Avin, Ilya Shpitser, and Judea Pearl. Identifiability of path-specific effects. In International Joint Conference on Artificial Intelligence, volume 19, pages 357-363, 2005.

[2] Judea Pearl. Causality: Models, Reasoning, and Inference. Cambridge University Press, 2 edition, 2009. ISBN 978-0521895606. 
[3] Thomas S. Richardson and Jamie M. Robins. Single world intervention graphs (SWIGs): A unification of the counterfactual and graphical approaches to causality. preprint: http: //www. csss. washington. edu/Papers/wp128. pdf, 2013.

[4] Jin Tian and Judea Pearl. On the testable implications of causal models with hidden variables. In Uncertainty in Artificial Intelligence, volume 18, pages 519-527. AUAI Press, 2002. 